\title{
From Completely Positive Maps to the Quantum Markovian Semigroup Master Equation
}

\author{
Daniel A. Lidar, ${ }^{1 *}$ Zsolt Bihary, ${ }^{2}$ and K. Birgitta Whaley ${ }^{1 \dagger}$ \\ ${ }^{1}$ Department of Chemistry, University of California, Berkeley, California 94720 \\ ${ }^{2}$ Department of Chemistry, University of California, Irvine, California 92697
}

\begin{abstract}
A central problem in the theory of the dynamics of open quantum systems is the derivation of a rigorous and computationally tractable master equation for the reduced system density matrix. Most generally, the evolution of an open quantum system is described by a completely positive linear map. We show how to derive a completely positive Markovian master equation (the Lindblad equation) from such a map by a coarse graining procedure. We provide a novel and explicit recipe for calculating the coefficients of the master equation, using perturbation theory in the weak-coupling limit. The only parameter external to our theory is the coarse-graining time-scale. We illustrate the method by explicitly deriving the master equation for the spin-boson model. The results are evaluated for the exactly solvable case of pure dephasing, and an excellent agreement is found within the timescale where the Markovian approximation is expected to be valid. The method can be extended in principle to include non-Markovian effects.
\end{abstract}

\section{INTRODUCTION}

The problem of the emergence of irreversible quantum dynamics from closed-system, unitary dynamics has occupied the attention of many researchers since the birth of quantum mechanics [1] 6]. It is generally believed that an acceptable solution is to view every quantum system as coupled to an environment, i.e., true quantum systems are always "open". The action of the environment is to perform measurements on the system, thus establishing a preferred "pointer basis" and leading to decoherence [7,8]. Within a Hamiltonian framework, recipes for deriving the associated reduced-dynamics were known since the early nineteen-sixties, starting with the Zwanzig projection technique: one writes down the Heisenberg equation of motion for the combined system-environment state, and then projects out the system by tracing out the environment degrees of freedom [9]. This yields an integro-differential equation involving an environment memory kernel, which must be subjected to approximations in order to become useful. The two main techniques available are the derivation of master equations by the use of the Born-Markov approximation [5], or a representation in terms of path integrals and influence functionals [6]. Unfortunately, in the former approach it is often unclear whether or not complete positivity is preserved in the sequence of approximations one makes [10], while in the latter approach one must make a semi-classical approximation in order to obtain a tractable theory [11].

Complete positivity in reduced dynamics is the very common-sensical idea ${ }^{1}$ that the open-system dynamics must preserve the positivity of a system's density matrix (a necessary condition for the probability interpreta- tion to hold) in the presence of any other non-interacting system. Building on this notion, two seminal contributions have been made. Kraus established an "operatorsum representation" which describes the most general completely-positive linear map on the density matrix of a quantum system [14]. This is a formal representation of the dynamics, which has been used profitably in the quantum information processing community 15., but is impractical to use for dynamics calculations. To address this, Lindblad has derived the most general completelypositive Markovian semigroup master equation for the dynamics of the density matrix 16]. This master equation can be integrated and solved to provide the timedevelopment of the system density matrix. Both of these results were derived on the basis of axiomatic quantum mechanics. While systematically satisfying, this approach nevertheless possesses the disadvantage that the resulting theories are necessarily phenomenological, in the sense that they contain no recipe for deriving their parameters from first principles [4].

Previous formal approaches therefore suffer from one of two disadvantages. Either the final equations are not necessarily completely positive, or they contain parameters which are not derived from first principles and must therefore be treated as phenomenological. In this paper we provide a derivation of the semigroup master equation (SME) from the Kraus operator-sum representation (OSR) which overcomes both of the above problems. Thus, the SME we derive is completely-positive (i.e., it is of Lindblad form), while we can also provide a recipe for calculating the parameters that appear in the equation. Our technique involves a coarse-graining procedure

\footnotetext{
${ }^{*}$ Current address: Department of Chemistry, 80 St. George Street, University of Toronto, Toronto, Ontario M5S 3H6

${ }^{\dagger}$ Corresponding author. Tel: 510-643-6820; fax: 510-643-1255; Email address:whaleysocrates.berkeley.edu.

${ }^{1}$ See [12,13] for a debate concerning this assertion.
} 
which leaves us with just one phenomenological parameter: the coarse-graining time-scale $\tau$. The work presented here is a continuation and generalization of [17], where the derivation of the SME from the OSR was provided for the first time. Here we verify the validity of the approach in 17, by proving now that the resulting SME is, as required, completely positive (Section II). Furthermore, we greatly expand the utility of the derivation by now also showing explicitly how to calculate the parameters that appear in the SME (Section III). The method uses a perturbative expansion in the system-bath coupling strength. We apply our formalism to the simple example of a collection of spins coupled to a boson bath, and compare the result to the exact solution (Section IV). We conclude with an overview and assessment of possible extensions (Section $\mathrm{V}$ ).

\section{FROM THE OPERATOR SUM REPRESENTATION OF REDUCED DYNAMICS TO THE SEMIGROUP MASTER EQUATION}

\section{A. Brief Review of the Operator Sum Representation}

The dynamics of a quantum system $S$ coupled to a bath $B$, which together form a closed system, evolves unitarily under the combined system-bath Hamiltonian

$$
\mathbf{H}_{S B}=\mathbf{H}_{S} \otimes \mathbf{I}_{B}+\mathbf{I}_{S} \otimes \mathbf{H}_{B}+\mathbf{H}_{I} .
$$

Here $\mathbf{H}_{S}, \mathbf{H}_{B}$ and $\mathbf{H}_{I}$ are, respectively, the system, bath and interaction Hamiltonians, and $\mathbf{I}$ is the identity operator. Assuming that $S$ and $B$ are initially decoupled, so that the total initial density matrix is a tensor product of the system and bath density matrices ( $\rho$ and $\rho_{B}$ respectively), the system dynamics are described by the reduced density matrix:

$$
\rho(0) \longmapsto \rho(t)=\operatorname{Tr}_{B}\left[\mathbf{U}\left(\rho \otimes \rho_{B}\right) \mathbf{U}^{\dagger}\right]
$$

Here $\operatorname{Tr}_{B}$ is the partial trace over the bath and

$$
\mathbf{U}=\exp \left(-\frac{i}{\hbar} \mathbf{H}_{S B} t\right)
$$

By using a spectral decomposition for the bath, $\rho_{B}=$ $\sum_{\mu} \wp_{\mu}|\mu\rangle\langle\mu|$ (where $\sum_{\mu} \wp_{\mu}=1$ ), and introducing a basis $\{|n\rangle\}_{n=1}^{N}$ for the $N$-dimensional system Hilbert space $\mathcal{H}$, this can be rewritten in the OSR as 14 :

$$
\rho(t)=\sum_{i=0}^{K} \mathbf{A}_{i}(t) \rho(0) \mathbf{A}_{i}^{\dagger}(t),
$$

where the Kraus operators $\left\{\mathbf{A}_{i}\right\}$ have matrix elements given by [4]:

$$
\left[\mathbf{A}_{i}\right]_{m n}(t)=\sqrt{\wp_{\mu}}\langle m|\langle\mu|\mathbf{U}(t)| \nu\rangle| n\rangle ; \quad i=(\mu, \nu) .
$$

$K=N_{B}^{2}$, where $N_{B}$ is the number of bath degrees of freedom. Also, by unitarity of $\mathbf{U}$, one derives the normalization condition

$$
\sum_{i=0}^{K} \mathbf{A}_{i}^{\dagger} \mathbf{A}_{i}=\mathbf{I}_{S}
$$

which guarantees preservation of the trace of $\rho$ : $\operatorname{Tr}[\rho(t)]=\operatorname{Tr}\left[\sum_{i} \mathbf{A}_{i} \rho(0) \mathbf{A}_{i}^{\dagger}\right]=\operatorname{Tr}\left[\rho(0) \sum_{i} \mathbf{A}_{i}^{\dagger} \mathbf{A}_{i}\right]=$ $\operatorname{Tr}[\rho(0)]$.The Kraus operators belong to the HilbertSchmidt space $\mathcal{A}(\mathcal{H})$ (itself a Hilbert space) of bounded operators acting on the system Hilbert space, and are represented by $N \times N$ matrices, just like $\rho$.

\section{B. Fixed-Basis Form of the Operator Sum Representation}

While the OSR evolution equation, Eq. 2.4), is perfectly general, it presents two major difficulties: (i) It is an evolution equation, rather than a differential equation, which expresses $\rho(t)$ in terms of the initial condition and time-dependent operators. Calculating these is equivalent to diagonalizing the entire system-bath Hamiltonian. This is impractical in all but a very few exactly solvable models. (ii) It is not clear how to separate out the unitary evolution of the system from the possibly non-unitary one, which occurs from the coupling of the system to the bath and leads to decoherence. The reason is that in general, each Kraus operator will contain a contribution from both the unitary and the non-unitary components of the evolution. When one makes the assumption of Markovian dynamics, however, as in the semigroup master equation (SME) [Eqs. 2.12),(2.13) below], both of these problems are solved, i.e., one obtains a differential equation in which there is an explicit separation between terms leading to unitary and to non-unitary evolution. This provided the motivation in [17] to develop an alternative representation of the OSR in a form which approaches the form of the SME as much as is possible, without yet making any Markovian assumption. We provide only the main steps of this derivation here, and refer the interested reader to [17] for full details.

It is convenient for this purpose to introduce a fixed operator basis for $\mathcal{A}(\mathcal{H})$. Let $\left\{\mathbf{K}_{\alpha}\right\}_{\alpha=0}^{M}$, with $\mathbf{K}_{0}=\mathbf{I}$, be such a basis, so that the expansion of the Kraus operators is given by:

$$
\mathbf{A}_{i}(t)=\sum_{\alpha=0}^{M} b_{i \alpha}(t) \mathbf{K}_{\alpha}
$$

Under this expansion, the OSR evolution equation, Eq. (2.4), becomes

$$
\rho(t)=\sum_{\alpha, \beta=0}^{M} \chi_{\alpha \beta}(t) \mathbf{K}_{\alpha} \rho(0) \mathbf{K}_{\beta}^{\dagger},
$$


where $\chi_{\alpha \beta}(t)$ is the matrix with elements

$$
\chi_{\alpha \beta}(t)=\sum_{i=0}^{K} b_{i \alpha}(t) b_{i \beta}^{*}(t) .
$$

The matrix $\chi$ is clearly Hermitian, with positive diagonal elements. With some algebraic manipulation [17] one can transform Eq. (2.8) into:

$$
\begin{aligned}
\frac{\partial \rho(t)}{\partial t}= & -\frac{i}{\hbar}[\dot{\mathbf{S}}(t), \rho(0)]+\frac{1}{2} \sum_{\alpha, \beta=1}^{M} \dot{\chi}_{\alpha \beta}(t) \times \\
& \left(\left[\mathbf{K}_{\alpha}, \rho(0) \mathbf{K}_{\beta}^{\dagger}\right]+\left[\mathbf{K}_{\alpha} \rho(0), \mathbf{K}_{\beta}^{\dagger}\right]\right) .
\end{aligned}
$$

where $\mathbf{S}(t)$ is the hermitian operator defined by

$$
\mathbf{S}(t)=\frac{i \hbar}{2} \sum_{\alpha=1}^{M}\left[\chi_{\alpha 0}(t) \mathbf{K}_{\alpha}-\chi_{0 \alpha}(t) \mathbf{K}_{\alpha}^{\dagger}\right] .
$$

Eq. (2.10) is the desired result: it represents a fixed-basis OSR evolution equation, with a strong resemblance to the SME, as we now detail.

\section{From the Fixed-Basis Operator Sum Representation Equation to the Semigroup Master Equation}

\section{Derivation of the Semigroup Master Equation by a Coarse Graining Procedure}

We recall that in the semigroup approach, under the assumptions of (i) Markovian dynamics, (ii) initial decoupling between the system and the bath, and (iii) the requirement of complete positivity, the system evolves according to the SME [4, 16]:

$$
\begin{aligned}
\frac{\partial \rho(t)}{\partial t} & =\mathrm{L}[\rho(t)] \equiv-\frac{i}{\hbar}\left[\mathbf{H}_{S}, \rho(t)\right]+\mathrm{L}_{D}[\rho(t)] \\
\mathrm{L}_{D}[\rho(t)] & =\frac{1}{2} \sum_{\alpha, \beta=1}^{M} a_{\alpha \beta}\left(\left[\mathbf{F}_{\alpha}, \rho(t) \mathbf{F}_{\beta}^{\dagger}\right]+\left[\mathbf{F}_{\alpha} \rho(t), \mathbf{F}_{\beta}^{\dagger}\right]\right),
\end{aligned}
$$

where $a_{\alpha \beta}$ is a constant positive semi-definite matrix. This equation bears a clear resemblance to Eq. (2.10). Analyzing the differences between the SME Eq. (2.13) and this OSR evolution equation (2.10) allows one to understand the precise manner in which the semigroup evolution arises from the OSR evolution under the above mentioned three conditions. An important difference between these two equations is the fact that the SME provides a prescription for determining $\rho(t)$ at all times $t$, given $\rho\left(t^{\prime}\right)$ as an initial condition at any other time $t>t^{\prime} \geq 0$, whereas Eq. (2.10) determines $\rho(t)$ in terms of $\rho(0)$, i.e., at the special time $t=0$ where the system and the bath are in a product state.
In [17] a coarse-graining procedure was introduced which allows to transform the exact Eq. (2.10) to the approximate SME. For convenience we repeat and clarify the derivation here. We consider three time-scales: (i) the inverse of the bath density of states frequency-cutoff $\tau_{c}$, (ii) a coarse-graining time-scale $\tau$ which is essentially the time-scale for the bath's "memory" to disappear (the definition will be made more precise below), and (iii) a system time-scale $\theta$ which is the typical time-scale for changes in the system density-matrix in the frame rotating with the system Hamiltonian. We require that

$$
\tau_{c} \ll \tau \ll \theta,
$$

and course-grain the evolution of the system in terms of $\tau: \rho_{j}=\rho(j \tau) ; \chi_{\alpha \beta ; j}=\chi_{\alpha \beta}(j \tau), j$ an integer. Further, rewriting the OSR Eq. (2.10) as $\rho(t)=\boldsymbol{\Lambda}(t) \rho(0)$ and defining $\tilde{\mathrm{L}}(t)$ through $\boldsymbol{\Lambda}(t)=\mathrm{T} \exp \left[\int_{0}^{t} \tilde{\mathrm{L}}(s) d s\right](\mathrm{T}$ indicates time-ordering) we have

$$
\frac{\partial \rho(t)}{\partial t}=\tilde{\mathrm{L}}(t)[\rho(t)]
$$

Define $\tilde{\mathrm{L}}_{j}=\int_{j \tau}^{(j+1) \tau} \tilde{\mathrm{L}}(s) d s$, with $\tau n=t: \int_{0}^{t} \tilde{\mathrm{L}}(s) d s=$ $\tau \sum_{j=0}^{n-1} \tilde{\mathrm{L}}_{j}$. Next we make the assumption that on the coarse-graining time-scale $\tau$, the evolution generators $\tilde{\mathrm{L}}(t)$ commute in the "average" sense that $\left[\tilde{\mathrm{L}}_{j}, \tilde{\mathrm{L}}_{k}\right]=$ $0, \forall j, k$. Physically, we imagine this operation as arising from the "resetting" of the bath density operator over the time-scale $\tau$. This means that $\tau$ must be larger than any characteristic bath time-scale, and explains the requirement $\tau_{c} \ll \tau$. Under this assumption, the evolution of the system is Markovian when $t \gg \tau: \boldsymbol{\Lambda}(t)=$ $\prod_{j=0}^{n-1} \exp \left[\tau \tilde{\mathrm{L}}_{j}\right]$. Further, under the discretization of the evolution, this product form of the evolution implies that $\rho_{j+1}=\exp \left[\tau \tilde{\mathrm{L}}_{j}\right]\left[\rho_{j}\right]$. In the limit of $\tau \ll t$ we expand this exponential, to find that

$$
\frac{\rho_{j+1}-\rho_{j}}{\tau}=\tilde{\mathrm{L}}_{j}\left[\rho_{j}\right] .
$$

This equation is simply a discretization of Eq. (2.15) under the assumption that $\tau \ll \theta$, where $\theta$ is the time-scale of change for the system density matrix. Notice in particular that the RHS of Eq. (2.16) contains the average value of $\tilde{\mathrm{L}}(t)$ over the interval. Now, from the OSR evolution equation (2.10), we know the explicit form of $\tilde{\mathrm{L}}(t)$ over the first interval from 0 to $\tau$. Discretizing over this interval we find that

$$
\begin{aligned}
\frac{\rho_{1}-\rho_{0}}{\tau} & =-\frac{i}{\hbar}\left[\langle\dot{\mathbf{S}}\rangle, \rho_{0}\right] \\
& +\frac{1}{2} \sum_{\alpha, \beta=1}^{M}\left\langle\dot{\chi}_{\alpha \beta}\right\rangle\left(\left[\mathbf{K}_{\alpha}, \rho_{0} \mathbf{K}_{\beta}^{\dagger}\right]+\left[\mathbf{K}_{\alpha} \rho(0), \mathbf{K}_{\beta}^{\dagger}\right]\right) \\
& \equiv \tilde{\mathrm{L}}_{0}\left[\rho_{0}\right]
\end{aligned}
$$

where 


$$
\langle X\rangle \equiv \frac{1}{\tau} \int_{0}^{\tau} X(s) d s .
$$

Thus, in the sense of the course graining above we have arrived at an explicit form for $\tilde{\mathrm{L}}_{0}$. However, deriving an explicit form for $\tilde{\mathrm{L}}_{1}$ and for higher terms beyond this first interval is impossible because Eq. (2.10) gives the evolution in terms of $\rho(0)$. Since we have made the assumption that the bath "resets" over the time-scale $\tau$, we expect the bath to interact with the system in the same manner over every $\tau$-length coarse-grained interval. This is equivalent to assuming that $\tilde{\mathrm{L}}_{i}=\tilde{\mathrm{L}}_{0}, \forall i$ (which of course is the most trivial way of satisfying the Markovian evolution condition $\left.\left[\tilde{\mathrm{L}}_{i}, \tilde{\mathrm{L}}_{j}\right]=0, \forall i, j\right)$. Then, under the natural identification of the K's with the $\mathbf{F}$ 's of the SME, and using Eq. (2.16), one is led to the well known form of the semigroup equation of motion:

$$
\begin{aligned}
\frac{\partial \rho(t)}{\partial t}= & -\frac{i}{\hbar}[\langle\dot{\mathbf{S}}\rangle, \rho(t)] \\
& +\frac{1}{2} \sum_{\alpha, \beta=1}^{M}\left\langle\dot{\chi}_{\alpha \beta}\right\rangle\left(\left[\mathbf{K}_{\alpha}, \rho(t) \mathbf{K}_{\beta}^{\dagger}\right]+\left[\mathbf{K}_{\alpha} \rho(t), \mathbf{K}_{\beta}^{\dagger}\right]\right)
\end{aligned}
$$

\section{Positivity of the Coefficient Matrix}

The positive semi-definiteness of the coefficient matrix $a_{\alpha \beta}$ in Eq. (2.13) is a sufficient condition for the preservation of complete positivity of the system dynamics [4. Thus, to complete the identification of Eq. (2.19) as a Lindblad equation, it only remains to be shown that $\left\langle\dot{\chi}_{\alpha \beta}\right\rangle$ is positive semi-definite. To do so let us show first that $\chi_{\alpha \beta}$ itself is positive semi-definite, i.e., that for any vector $\mathbf{c}$, the matrix $\chi$ satisfies $\mathbf{c} \chi \mathbf{c}^{* t} \geq 0$ :

$$
\begin{aligned}
\mathbf{c} \chi \mathbf{c}^{* t} & =\sum_{\alpha \beta} c_{\alpha}^{*} \chi_{\alpha \beta} c_{\beta}=\sum_{i, \alpha \beta} c_{\alpha}^{*} b_{i \alpha} b_{i \beta}^{*} c_{\beta} \\
& =\sum_{i}\left|\sum_{\alpha} c_{\alpha}^{*} b_{i \alpha}\right|^{2} \geq 0
\end{aligned}
$$

where we used Eq. (2.9). Next,

$$
\left\langle\dot{\chi}_{\alpha \beta}\right\rangle=\frac{1}{\tau} \int_{0}^{\tau} \dot{\chi}_{\alpha \beta} d t=\frac{1}{\tau}\left(\chi_{\alpha \beta}(\tau)-\chi_{\alpha \beta}(0)\right),
$$

so that we must show that $\chi(0)$ does not spoil the positivity. Now, from Eqs. (2.3) and (2.5) $\mathbf{A}_{i}(0)=$ $\sqrt{\wp_{\mu}}\langle\mu|\mathbf{U}(0)| \nu\rangle=\sqrt{\wp_{\mu}} \delta_{\mu \nu} \mathbf{I}_{S}=\sum_{\alpha=0}^{M} b_{i \alpha}(0) \mathbf{K}_{\alpha}$, so that $b_{i \alpha}(0)=\sqrt{\wp_{\mu}} \delta_{\alpha 0} \delta_{i,(\mu, \mu)}\left(\right.$ recall $\left.\mathbf{K}_{0}=\mathbf{I}_{S}\right)$. Thus

$$
\begin{aligned}
\chi_{\alpha \beta}(0) & =\sum_{i} b_{i \alpha}(0) b_{i \beta}^{*}(0)=\sum_{i=(\mu, \nu)} \wp_{\mu} \delta_{\alpha 0} \delta_{\beta 0} \delta_{i,(\mu, \mu)} \\
& =\delta_{\alpha 0} \delta_{\beta 0} .
\end{aligned}
$$

But in Eq. (2.19) we are concerned with $\left\langle\dot{\chi}_{\alpha \beta}\right\rangle$ only for $\alpha, \beta \geq 1$, so that finally, from Eq. (2.21), the submatrix $\left\langle\dot{\chi}_{\alpha \beta}\right\rangle$ with $\alpha, \beta \geq 1$ is indeed positive semi-definite. The important conclusion is that Eq. (2.19) is in Lindblad form, i.e., it preserves complete positivity. This establishes the validity of our result for the SME, and should be contrasted with projection-operator type derivations of the master equation [5, 18], which do not necessarily satisfy the complete positivity criterion.

\section{Separating Out the Hamiltonian}

We can write Eq. (2.19) in an alternative form which distinguishes between the system and bath contributions to the unitary part of the evolution. Because Eq. 2.10) is linear in the $\chi_{\alpha \beta}(t)$ matrix, one can calculate $\chi_{\alpha \beta}^{(0)}(t)$ for the isolated system and hence define the new terms which come about from the coupling of the system to the bath: $\chi_{\alpha \beta}(t)=\chi_{\alpha \beta}^{(0)}(t)+\chi_{\alpha \beta}^{(1)}(t)$. The terms which correspond to the isolated system will then produce a normal $-(i / \hbar)\left[\mathbf{H}_{S}, \rho(t)\right]$ Liouville term in Eq. (2.19). Thus Eq. (2.19) can be rewritten as

$$
\begin{aligned}
\frac{\partial \rho(t)}{\partial t}= & -\frac{i}{\hbar}\left[\mathbf{H}_{S}+\left\langle\dot{\mathbf{S}}^{(1)}\right\rangle, \rho(t)\right] \\
& +\frac{1}{2} \sum_{\alpha, \beta=1}^{M}\left\langle\dot{\chi}_{\alpha \beta}\right\rangle\left(\left[\mathbf{K}_{\alpha}, \rho(t) \mathbf{K}_{\beta}^{\dagger}\right]+\left[\mathbf{K}_{\alpha} \rho(t), \mathbf{K}_{\beta}^{\dagger}\right]\right) .
\end{aligned}
$$

Identifying $\left\langle\dot{\chi}_{\alpha \beta}\right\rangle$ with $a_{\alpha \beta}$, and $\mathbf{K}_{\alpha}$ with $\mathbf{F}_{\alpha}$, this is seen to be equivalent to Eqs.(2.12)-(2.13), except for the presence of the second term in the Liouvillian. This second term $\left\langle\dot{\mathbf{S}}^{(1)}\right\rangle$, inducing unitary dynamics on the system, is referred to as the Lamb shift. It explicitly describes the effect the bath has on the unitary part of the system dynamics, and "renormalizes" the system Hamiltonian. It is often implicitly assumed to be present in Eq. (2.12) 19.

In summary, we have shown in this Section how coarsegraining the evolution over the bath memory time-scale $\tau$ allows one to understand the connection between the OSR evolution and the semigroup dynamics. The importance of Eq. (2.10) lies in the fact that it allows one to pinpoint the exact point at which the assumption of Markovian dynamics is made. Furthermore, due to the general likeness of its form to the SME, it provides an easily translatable connection from the non-Markovian OSR to the Markovian SME. Notice also that the assumption of Markovian dynamics introduces an arrow of time in the evolution of the system, through the ordering of the environmental states: The system evolves through time in the direction of successive resettings of the bath. Additionally, it is important to note that we have shown that our procedure leads to an explicitly Lindbladian (completely positive) form of the SME, as written in final form in Eq. (2.23). 
Finally, we address the question of the inclusion of nonMarkovian effects. The approach presented here also offers a route to a systematic inclusion of non-Markovian effects, i.e., higher order dynamics which include bath memory terms. Such a derivation of a "post-Markovian" master equation is a long sought-after goal of the field of open quantum systems. Several attempts have been reported, but generally the resulting equations are not satisfactory because complete positivity is violated [20]. In the context of the present approach, the formal extension to go beyond the Markovian regime can be made by replacing the assumption that the evolution generators $\tilde{\mathrm{L}}_{j}$ commute to first order [see text below Eq. (2.15)], by a higher order commutator. The derivation of this commutator and the resulting post-Markovian master equation is left to a future publication.

\section{EXPLICIT DERIVATION OF THE SEMIGROUP MASTER EQUATION PARAMETERS}

We can now exploit the coarse-grained first-order (in time) perturbation expansion of the OSR, Eq. (2.10), made in the previous section, in order to derive the $e x$ plicit form of the parameters and operators appearing in the resulting SME. To do so, it turns out to be most convenient to work in the interaction picture (IP) defined with respect to the free system and bath Hamiltonians. Let us the system-bath interaction Hamiltonian of Eq. (2.1) in the following perfectly general form: ${ }^{2}$

$$
\mathbf{H}_{I}=\sum_{\alpha} \lambda_{\alpha} \mathbf{S}_{\alpha} \otimes \mathbf{B}_{\alpha}
$$

where $\left\{\mathbf{S}_{\alpha}\right\}$ and $\left\{\mathbf{B}_{\alpha}\right\}$ are the system and bath operators respectively, and $\left\{\lambda_{\alpha}\right\}$ are coupling coefficients. In the IP we do not have to deal directly with the free system and bath Hamiltonians. However, as will be seen below, we do recover the Lamb shift.

\section{A. The Interaction Picture}

Transformation to the IP is accomplished by means of the unitary operator

$$
\mathbf{U}_{T}=\exp \left(-i t \mathbf{H}_{S}\right) \otimes \exp \left(-i t \mathbf{H}_{B}\right) \equiv \mathbf{U}_{S} \otimes \mathbf{U}_{B}
$$

\footnotetext{
${ }^{2}$ Note that $\left\{\mathbf{S}_{\alpha}\right\}$ and $\left\{\mathbf{B}_{\alpha}\right\}$ are not assumed to be linear operators, and that any interaction Hamiltonian can be decomposed into a sum of terms acting separately on system and bath. Furthermore, we allow $\left\{\mathbf{S}_{\alpha}\right\}$ and $\left\{\mathbf{B}_{\alpha}\right\}$ to be timedependent.
}

Operators in the IP will be denoted using explicit time dependence (and where there already was a time dependence, with an $I$ subscript). Thus:

$$
\mathbf{H}_{I}(t)=\mathbf{U}_{T}^{\dagger} \mathbf{H}_{I} \mathbf{U}_{T}=\sum_{\alpha} \lambda_{\alpha} \mathbf{S}_{\alpha}(t) \otimes \mathbf{B}_{\alpha}(t)
$$

where

$$
\begin{aligned}
& \mathbf{S}_{\alpha}(t)=\mathbf{U}_{S}^{\dagger} \mathbf{S}_{\alpha} \mathbf{U}_{S}=\sum_{\beta} p_{\alpha \beta}(t) \mathbf{S}_{\beta} \\
& \mathbf{B}_{\alpha}(t)=\mathbf{U}_{B}^{\dagger} \mathbf{B}_{\alpha} \mathbf{U}_{B}=\sum_{\beta} q_{\alpha \beta}(t) \mathbf{B}_{\beta},
\end{aligned}
$$

with $p_{\alpha \beta}(0)=q_{\alpha \beta}(0)=\delta_{\alpha \beta}$. The density matrix for the system and bath combined is denoted $\rho_{\text {tot }}(t)$ in the Schrödinger picture, and is transformed to the IP by $\rho_{\text {tot }, I}(t)=\mathbf{U}_{T}^{\dagger} \rho_{\text {tot }}(t) \mathbf{U}_{T}$. The dynamics of $\rho_{\text {tot }, I}(t)$ is governed by the unitary propagator $\mathbf{U}(t)=$ $\mathbf{U}_{T}^{\dagger} \exp \left(-i t \mathbf{H}_{S B}\right) \mathbf{U}_{T}$, where $\mathbf{H}_{S B}$ is the full systembath Hamiltonian: $\rho_{\text {tot }, I}(t)=\mathbf{U}(t) \rho_{\text {tot }, I}(0) \mathbf{U}^{\dagger}(t)$. The Schrödinger and interaction pictures coincide at $t=0$ so that $\rho_{\text {tot }, I}(0)=\rho_{\text {tot }}(0)=\rho(0) \otimes \rho_{B}(0)$. It is a standard exercise to show that 21]

$$
\begin{aligned}
\mathbf{U}(t) & =\mathrm{T} \exp \left[-\frac{i}{\hbar} \int_{0}^{t} \mathbf{H}_{I}(\tau) d \tau\right] \\
& =\mathbf{I}+\sum_{n=1}^{\infty} \frac{(-i)^{n}}{n !} \mathbf{U}_{n}(t)
\end{aligned}
$$

where

$\mathbf{U}_{n}(t) \equiv \int_{0}^{t} d t_{n} \int_{0}^{t} d t_{n-1} \cdots \int_{0}^{t} d t_{1} \mathrm{~T}\left\{\mathbf{H}_{I}\left(t_{1}\right) \cdots \mathbf{H}_{I}\left(t_{n}\right)\right\}$

The Dyson time-ordered product is defined with respect to any set of operators $\mathbf{O}_{i}\left(t_{\tau}\right)$ as [21]: $\mathrm{T}\left\{\mathbf{O}_{1}\left(t_{1}\right) \cdots \mathbf{O}_{n}\left(t_{n}\right)\right\}=\mathbf{O}_{\tau_{1}}\left(t_{\tau_{1}}\right) \cdots \mathbf{O}_{\tau_{n}}\left(t_{\tau_{n}}\right)$, where $t_{\tau_{1}}>t_{\tau_{2}}>\ldots>t_{\tau_{n}}$. The system density matrix in the IP is obtained, as usual, by tracing over the bath, which leads to the OSR:

$$
\rho_{I}(t)=\operatorname{Tr}_{B}\left[\rho_{\mathrm{tot}, I}(t)\right]=\sum_{i=0}^{K} \mathbf{A}_{i}(t) \rho(0) \mathbf{A}_{i}^{\dagger}(t),
$$

where the Kraus operators are now defined in the IP:

$$
\mathbf{A}_{i}(t)=\sqrt{\wp_{\mu}}\langle\mu|\mathbf{U}(t)| \nu\rangle
$$


Repeating the derivation of Sections II B, II C we thus obtain the very same form for the SME as in Eq. (2.19), but now it is a SME for the interaction representation, $\rho_{I}(t)$. Finally, the transformation back to the Schrödinger picture is accomplished by:

$$
\rho(t)=\mathbf{U}_{S} \rho_{I}(t) \mathbf{U}_{S}^{\dagger} .
$$

\section{B. Perturbation Theory Expansion of the Kraus Operators}

Our next task is to calculate the Kraus operators. We do so by using the expansion for $\mathbf{U}(t)$ and Eqs. (3.6), (3.9). We have then:

$$
\mathbf{A}_{i}(t)=\sqrt{\wp_{\mu}} \delta_{\mu \nu} \mathbf{I}_{S}+\sum_{n=1}^{\infty} \mathbf{A}_{i}^{(n)}(t)
$$

where

$$
\begin{aligned}
\mathbf{A}_{i}^{(n)}(t)= & \sqrt{\wp_{\mu}} \frac{(-i)^{n}}{n !} \sum_{\alpha_{1}, \ldots, \alpha_{n}} \int_{0}^{t} d t_{n} \int_{0}^{t} d t_{n-1} \ldots \int_{0}^{t} d t_{1} \times \\
\mathrm{T} & {\left[\prod_{j=1}^{n} \lambda_{\alpha_{j}} \mathbf{S}_{\alpha_{j}}\left(t_{j}\right)\right]\left\langle\mu\left|\mathrm{T}\left[\prod_{j=1}^{n} \mathbf{B}_{\alpha_{j}}\left(t_{j}\right)\right]\right| \nu\right\rangle, }
\end{aligned}
$$

and we used $\left[\mathbf{S}_{\alpha_{i}}\left(t_{i}\right), \mathbf{B}_{\alpha_{j}}\left(t_{j}\right)\right]=0$ to separate the timeordering operations. $\mathbf{A}_{i}^{(n)}$ is proportional to $\left(\lambda_{\alpha}\right)^{n}$, so that in the weak-coupling case of $\lambda_{\alpha} \ll 1$, we can truncate the expansion at small $n$.

\section{First Order Case}

First we note that from Eq. (3.11), with $\mathbf{K}_{0}=\mathbf{I}_{S}$ : $b_{i 0}(t)=\sqrt{\wp_{\mu}} \delta_{\mu \nu}$. Now, let us calculate the expression for $n=1$. In this case there is no need to worry about time ordering, and we have:

$$
\begin{aligned}
\mathbf{A}_{i=\mu \nu}^{(1)}(t) & =-i \sqrt{\wp \mu} \sum_{\beta} \int_{0}^{t} d t_{1} \lambda_{\beta} \mathbf{S}_{\beta}\left(t_{1}\right)\left\langle\mu\left|\mathbf{B}_{\beta}\left(t_{1}\right)\right| \nu\right\rangle \\
& =-i t \sqrt{\wp_{\mu}} \sum_{\alpha \beta \gamma} \mathbf{S}_{\alpha} \lambda_{\beta}\left\langle\mu\left|\mathbf{B}_{\gamma}\right| \nu\right\rangle \Gamma_{\beta}^{\alpha \gamma}(t) \\
& \equiv \sum_{\alpha} b_{i \alpha}(t) \mathbf{K}_{\alpha}
\end{aligned}
$$

where the second line follows using Eqs. (3.4), 3.5) the third from the fixed-basis operator expansion in Eq. (2.7), and we defined

$$
\Gamma_{\alpha}^{\beta \gamma}(t) \equiv \frac{1}{t} \int_{0}^{t} d t_{1} p_{\alpha \beta}\left(t_{1}\right) q_{\alpha \gamma}\left(t_{1}\right)
$$

This dimensionless quantity thus depends entirely on the transformation to the interaction picture, i.e., it contains no information on the system-bath coupling, but only on the internal system and bath dynamics. Next, let us identify $\mathbf{K}_{\alpha}=\mathbf{S}_{\alpha}$ (the system operators) and assume that our basis is trace-orthogonal:

$$
\operatorname{Tr}\left[\mathbf{S}_{\alpha}^{\dagger} \mathbf{S}_{\beta}\right]=\delta_{\alpha \beta} / N_{\alpha}
$$

where $N_{\alpha}$ is a normalization constant. Then

$$
b_{i \alpha}(t)=-i t \sqrt{\wp_{\mu}} \sum_{\alpha^{\prime} \alpha^{\prime \prime}} \lambda_{\alpha^{\prime}}\left\langle\mu\left|\mathbf{B}_{\alpha^{\prime \prime}}\right| \nu\right\rangle \Gamma_{\alpha^{\prime}}^{\alpha \alpha^{\prime \prime}}(t) \quad \alpha \geq 1 .
$$

Using these results and $\chi_{\alpha \beta}(t)=\sum_{i=\mu \nu} b_{i \alpha}(t) b_{i \beta}^{*}(t)$ we can reconstruct the $\chi$ matrix: $\chi_{00}(t)=\sum_{\mu} \wp_{\mu}=1$, and for $\alpha \geq 1$ :

$$
\begin{aligned}
\chi_{\alpha 0}(t) & =\sum_{i=\mu \nu} b_{i \alpha}(t) b_{i 0}^{*}(t)=\sum_{\mu} \sqrt{\wp_{\mu}} b_{\mu \mu, \alpha}(t) \\
& =-i t \sum_{\alpha^{\prime} \alpha^{\prime \prime}} \lambda_{\alpha^{\prime}}\left\langle\mathbf{B}_{\alpha^{\prime \prime}}\right\rangle_{B} \Gamma_{\alpha^{\prime}}^{\alpha \alpha^{\prime \prime}}(t)
\end{aligned}
$$

where we used

$$
\langle\mathbf{X}\rangle_{B} \equiv \operatorname{Tr}\left[\rho_{B} \mathbf{X}\right]=\sum_{\mu} \wp_{\mu}\langle\mu|\mathbf{X}| \mu\rangle
$$

defining the bath-averaged expectation value of an arbitrary operator $\mathbf{X}$. Finally, for both $\alpha, \beta \geq 1$

$$
\begin{aligned}
\chi_{\alpha \beta}(t) & =\sum_{i=\mu \nu} b_{i \alpha}(t) b_{i \beta}^{*}(t) \\
& =t^{2} \sum_{\alpha^{\prime} \alpha^{\prime \prime} \beta^{\prime} \beta^{\prime \prime}} \lambda_{\alpha^{\prime}} \lambda_{\beta^{\prime}}^{*}\left\langle\mathbf{B}_{\alpha^{\prime \prime}} \mathbf{B}_{\beta^{\prime \prime}}^{\dagger}\right\rangle_{B} \Gamma_{\alpha^{\prime}}^{\alpha \alpha^{\prime \prime}}(t)\left(\Gamma_{\beta^{\prime}}^{\beta \beta^{\prime \prime}}(t)\right)^{*}
\end{aligned}
$$

where in the last line we used the completeness relation $\sum_{\nu}|\nu\rangle\langle\nu|=\mathbf{I}_{B}$

Now, as shown in Eqs. 2.21), (2.22):

$$
\begin{aligned}
a_{\alpha \beta} & =\left\langle\dot{\chi}_{\alpha \beta}\right\rangle=\frac{1}{\tau}\left(\chi_{\alpha \beta}(\tau)-\chi_{\alpha \beta}(0)\right) \\
& =\frac{\chi_{\alpha \beta}(\tau)}{\tau}
\end{aligned}
$$

unless both $\alpha=\beta=0$ (in which case $\left\langle\dot{\chi}_{00}\right\rangle=0$ ). Together with the SME Eq. (2.19) we thus have all the ingredients. In particular, we can calculate the $\langle\dot{\mathbf{S}}\rangle$ Lamb shift term in Eq. (2.19) from Eq. 2.11):

$$
\begin{aligned}
\langle\dot{\mathbf{S}}\rangle & =\frac{i}{2} \sum_{\alpha}\left\langle\dot{\chi}_{\alpha 0}\right\rangle \mathbf{S}_{\alpha}-\left\langle\dot{\chi}_{\alpha 0}\right\rangle^{*} \mathbf{S}_{\alpha}^{\dagger} \\
& =\frac{1}{2} \sum_{\alpha} \phi_{\alpha} \mathbf{S}_{\alpha}+\phi_{\alpha}^{*} \mathbf{S}_{\alpha}^{\dagger},
\end{aligned}
$$

where 


$$
\phi_{\alpha} \equiv \sum_{\alpha^{\prime} \alpha^{\prime \prime}} \lambda_{\alpha^{\prime}}\left\langle\mathbf{B}_{\alpha^{\prime \prime}}\right\rangle_{B} \Gamma_{\alpha^{\prime}}^{\alpha \alpha^{\prime \prime}}(\tau)
$$

is a correlation function which contributes to the Lamb shift. Note that unlike in Eq. 2.23), $\langle\dot{\mathbf{S}}\rangle$ does not contain the system Hamiltonian, as indeed it should not in the IP.

As for the decoherence term, we find

$$
\begin{aligned}
a_{\alpha \beta} & =\left\langle\dot{\chi}_{\alpha \beta}\right\rangle \\
& =\tau \sum_{\alpha^{\prime} \alpha^{\prime \prime} \beta^{\prime} \beta^{\prime \prime}} \lambda_{\alpha^{\prime}} \lambda_{\beta^{\prime}}^{*}\left\langle\mathbf{B}_{\alpha^{\prime \prime}} \mathbf{B}_{\beta^{\prime \prime}}^{\dagger}\right\rangle_{B} \Gamma_{\alpha^{\prime}}^{\alpha \alpha^{\prime \prime}}(\tau)\left(\Gamma_{\beta^{\prime}}^{\beta \beta^{\prime \prime}}(\tau)\right)^{*} .
\end{aligned}
$$

Note that both the Lamb shift parameters $\phi_{\alpha}$ and the decoherence parameters $a_{\alpha \beta}$ formally depend on the coarsegraining time $\tau$. Since $\tau$ is a "dummy" differentiation parameter in our theory, the dependence upon it should disappear in an explicit calculation. We deal with this in the example studied in Section IV.

\section{Second Order Case}

Expanding the Kraus operators to second order yields:

$$
\begin{aligned}
\mathbf{A}_{i=\mu \nu}^{(2)}(t)= & -\frac{\sqrt{\wp_{\mu}}}{2} \sum_{\alpha_{1}, \alpha_{2}} \lambda_{\alpha_{1}} \lambda_{\alpha_{2}} \int_{0}^{t} d t_{2} \int_{0}^{t} d t_{1} \times \\
& \mathrm{T}\left[\mathbf{S}_{\alpha_{1}}\left(t_{1}\right) \mathbf{S}_{\alpha_{2}}\left(t_{2}\right)\right]\left\langle\mu\left|\mathrm{T}\left[\mathbf{B}_{\alpha_{1}}\left(t_{1}\right) \mathbf{B}_{\alpha_{2}}\left(t_{2}\right)\right]\right| \nu\right\rangle \\
= & -\frac{\sqrt{\wp_{\mu}} t^{2}}{2} \sum_{\alpha_{1} \alpha_{2}} \mathbf{S}_{\alpha_{1}} \mathbf{S}_{\alpha_{2}} \times \\
& \sum_{\beta_{1} \beta_{2}, \gamma_{1} \gamma_{2}} \lambda_{\beta_{1}} \lambda_{\beta_{2}}\left\langle\mu\left|\mathbf{B}_{\gamma_{1}} \mathbf{B}_{\gamma_{2}}\right| \nu\right\rangle \Gamma_{\beta_{1} \beta_{2}}^{\alpha_{1} \alpha_{2} ; \gamma_{1} \gamma_{2}}(t)
\end{aligned}
$$

where

$$
\begin{aligned}
& \Gamma_{\alpha_{1} \alpha_{2}}^{\beta_{1} \beta_{2} ; \gamma_{1} \gamma_{2}}(t) \equiv \frac{1}{t^{2}} \int_{0}^{t} d t_{2} \int_{0}^{t} d t_{1} \mathrm{~T}\left[p_{\alpha_{1} \beta_{1}}\left(t_{1}\right) p_{\alpha_{2} \beta_{2}}\left(t_{2}\right)\right] \times \\
& \mathrm{T}\left[q_{\alpha_{1} \gamma_{1}}\left(t_{1}\right) q_{\alpha_{2} \gamma_{2}}\left(t_{2}\right)\right]
\end{aligned}
$$

We need to compare this expression to the expansion of the Kraus operators in terms of the fixed basis, $\mathbf{A}_{i}(t)=$ $\sum_{\alpha=0}^{M} b_{i \alpha}(t) \mathbf{K}_{\alpha}$ [Eq. 2.7)]. To do so we must now extend the fixed basis set so that it includes product terms:

$$
\sum_{\alpha=0}^{M} b_{i \alpha}(t) \mathbf{K}_{\alpha}=\sum_{\alpha_{1}=0} b_{i ; \alpha_{1}}(t) \mathbf{S}_{\alpha_{1}}+\sum_{\alpha_{1}, \alpha_{2}=0} b_{i ; \alpha_{1} \alpha_{2}}(t) \mathbf{S}_{\alpha_{1}} \mathbf{S}_{\alpha_{2}}
$$

Comparing this expansion to Eq. (3.24) we can read off the second order time-dependent coefficients $b_{i ; \alpha_{1} \alpha_{2}}$ as

$$
b_{i ; \alpha_{1} \alpha_{2}}=-\frac{\sqrt{\wp_{\mu}} t^{2}}{2} \sum_{\beta_{1} \beta_{2}, \gamma_{1} \gamma_{2}} \lambda_{\beta_{1}} \lambda_{\beta_{2}}\left\langle\mu\left|\mathbf{B}_{\gamma_{1}} \mathbf{B}_{\gamma_{2}}\right| \nu\right\rangle \Gamma_{\beta_{1} \beta_{2}}^{\alpha_{1} \alpha_{2} ; \gamma_{1} \gamma_{2}}(t) \text {, }
$$

provided that the basis of system operators is closed under multiplication (or more generally: is traceorthonormal for all products of basis elements). The decoherence and Lamb shift parameters can then be calculated as in the first-order case.

\section{E. Putting It All Together}

In conclusion, we have derived an explicit form for the SME of Eq. (2.19) (with $\mathbf{S}_{\alpha}$ replacing $\mathbf{K}_{\alpha}$ ). To find the full SME for a given problem, it is necessary to:

1. Identify the system operators $\left\{\mathbf{S}_{\alpha}\right\}$ in the interaction Hamiltonian (and recall that these operators must be trace-orthogonal in our formalism).

2. Solve for the time-dependent system and bath operators in the interaction picture [Eqs. (3.4), 3.5)], and thus find $\Gamma$ from Eq. (3.14).

3. Calculate the expectation values of the bath operators. The results of this step will depend on the initial state of the bath (e.g., thermal equilibrium, coherent state, squeezed state, etc.).

4. Use the results of the previous steps to calculate the Lamb shift term $\langle\dot{\mathbf{S}}\rangle$, the decoherence matrix $\left\langle\dot{\chi}_{\alpha \beta}\right\rangle$, and finally, to write down the SME.

Since this SME is of the Lindblad form [16] it is guaranteed to preserve positivity of the density matrix. Systematic corrections may be derived by continuing the expansion in Eq. (3.11) to higher orders in $n$.

\section{EXAMPLE: SPIN-BOSON MODEL}

\section{A. Pure Dephasing of Multiple Qubits}

As a concrete and simple example of the procedure described above, we consider the form of the SME derived for a collection of independent two-level systems (qubits) coupled via a phase-damping (non-dissipative) interaction to a boson bath. The Hamiltonians in this spin-boson model are [22]:

$$
\begin{aligned}
\mathbf{H}_{S} & =-\frac{1}{2} \sum_{i} \hbar \omega_{0}^{i} \sigma_{z}^{i}, \\
\mathbf{H}_{B} & =\sum_{k} \hbar \omega_{k}\left(\mathbf{N}_{k}+\frac{1}{2}\right), \\
\mathbf{H}_{I} & =\sum_{i, k} \sigma_{z}^{i} \otimes\left(\lambda_{k}^{i} \mathbf{b}_{k}+\lambda_{k}^{i *} \mathbf{b}_{k}^{\dagger}\right),
\end{aligned}
$$


where $\hbar \omega_{0}^{i}$ are the qubit energies, $\lambda_{k}^{i}$ are coupling coefficients, $\mathbf{b}_{k}$ and $\mathbf{b}_{k}^{\dagger}$ are the $k^{\text {th }}$ bath mode annihilation and creation operators obeying the boson commutation relation $\left[\mathbf{b}_{k}, \mathbf{b}_{l}^{\dagger}\right]=\mathbf{1} \delta_{k l}$, and $\mathbf{N}_{k}=\mathbf{b}_{k}^{\dagger} \mathbf{b}_{k}$ is the number operator. Comparing to Eq. (3.1) we read off the system operators as $\mathbf{S}_{\alpha}=\sigma_{z}^{i}$, and the bath operators as $\mathbf{B}_{\alpha}=\mathbf{b}_{k}$. Below we deal with the required modifications to our treatment of the indices in order to account for these assignments.

We assume that the boson bath is in thermal equilibrium at temperature $T=1 /\left(k_{B} \beta\right) \quad\left(k_{B}\right.$ is the Boltzman constant). Thus the bath density matrix is $\rho_{B}=\frac{1}{Z} e^{-\beta \mathbf{H}_{B}}=\frac{1}{Z} \sum_{\mu} e^{-\beta E_{\mu}}|\mu\rangle\langle\mu|$, where $\mu=\left\{n_{1}, n_{2}, \ldots, n_{k}, \ldots\right\}$ are the numbers of quanta in all bath modes, $E_{\mu}$ is the energy of the field at a given occupation $\mu$, and $Z(T)=\operatorname{Tr}\left[\exp \left(-\beta \mathbf{H}_{B}\right)\right]=$ $\prod_{k} e^{-\beta \hbar \omega_{k} / 2} /\left(1-e^{-\beta \hbar \omega_{k}}\right)$ is the canonical partition function. Some useful results for the average number of quanta in the $k^{\text {th }}$ bath mode and the averages of the creation and annihilation operators are:

$$
\begin{aligned}
\left\langle\mathbf{b}_{k}^{\dagger} \mathbf{b}_{l}\right\rangle_{B} & =\left\langle\mathbf{b}_{k} \mathbf{b}_{l}^{\dagger}\right\rangle-\delta_{k, l}=\delta_{k, l} \frac{1}{e^{\beta \hbar \omega_{k}}-1}, \\
\left\langle\mathbf{b}_{k}^{\dagger}\right\rangle_{B} & =\left\langle\mathbf{b}_{k}\right\rangle_{B}=\left\langle\mathbf{b}_{k}^{\dagger} \mathbf{b}_{l}^{\dagger}\right\rangle_{B}=\left\langle\mathbf{b}_{k} \mathbf{b}_{l}\right\rangle_{B}=0 .
\end{aligned}
$$

We now proceed to calculate the various quantities appearing in the SME.

\section{Form of the Interaction Representation Operators}

Formally, we need to solve Eqs. 3.4), (3.5) for the timedependent system and bath operators. In the present simple example, however it is clear that since $\sigma_{z}^{i}$ commutes with the system Hamiltonian, $\sigma_{z}^{i}(t)=\sigma_{z}^{i}(0) \equiv \sigma_{z}^{i}$. Further, it is an elementary exercise to show that $\mathbf{b}_{k}(t)=$ $\mathbf{b}_{k} e^{i \omega_{k} t}$. Therefore the interaction Hamiltonian in the IP is:

$$
\mathbf{H}_{I}(t)=\sum_{i, k} \sigma_{z}^{i} \otimes\left(\lambda_{k}^{i} e^{i \omega_{k} t} \mathbf{b}_{k}+\lambda_{k}^{i *} e^{-i \omega_{k} t} \mathbf{b}_{k}^{\dagger}\right) .
$$

\section{Calculation of the Lamb Shift and Decoherence Parameters}

In the derivation of Section $[I I C$ the interaction Hamiltonian was expressed as a sum over the single index $\alpha$, in order to reduce the clutter of indices to a minimum. However, as seen from the interaction Hamiltonian of Eq. (4.3), in reality we need more indices. In particular, we need to clarify the indexation of $\Gamma_{\alpha}^{\beta \gamma}$ of Eq. (3.14): each of the indices $\alpha, \beta$ and $\gamma$ may now correspond to a qubit position (denoted $i$ or $j$ ), a Pauli matrix index (denoted $\xi=x, y, z$ ), or a bath mode (denoted $k$ or $l$ ). Qubits variables can have both position and Pauli indices, but bath variables have only a mode index. We will use a comma to separate qubit and bath variables, as in $\alpha=(i \xi, k)$, when all three indices are needed. When one of the indices is irrelevant it will simply be dropped. To separate groups of indices, such as the $\beta \gamma$ in $\Gamma_{\alpha}^{\beta \gamma}$, we will use a semicolon. For example, $\Gamma_{i z, k}^{j \xi ;}$ is short for $\Gamma_{\alpha}^{\beta \gamma}$ with $\alpha=(i z, k), \beta=\left(j \xi, k^{\prime}\right), \gamma=\left(j^{\prime} \xi_{1}, l\right)$, where $k^{\prime}$, $j^{\prime}$ and $\xi_{1}$ are irrelevant. With these preparations we are now ready to calculate $\Gamma_{\alpha}^{\beta \gamma}$. By comparing $\sigma_{z}^{i}(t)=\sigma_{z}^{i}$, $\mathbf{b}_{k}(t)=\mathbf{b}_{k} e^{i \omega_{k} t}$ to Eqs. (3.4), 3.5) and using the correct index convention, we have that $p_{z \xi}^{i i^{\prime}}(t)=\delta_{i i^{\prime}} \delta_{\xi z}$ and $q_{k k^{\prime \prime}}(t)=\delta_{k k^{\prime \prime}} e^{i \omega_{k} t}$. Therefore:

$$
\begin{aligned}
\Gamma_{i z, k}^{i^{\prime} \xi ; k^{\prime \prime}}(t) & =\frac{1}{t} \int_{0}^{t} d t_{1} p_{z \xi}^{i i^{\prime}}\left(t_{1}\right) q_{k k^{\prime \prime}}\left(t_{1}\right) \\
& =\delta_{i i^{\prime}} \delta_{k k^{\prime \prime}} \delta_{\xi z} e^{i \omega_{k} t / 2} \operatorname{sinc} \frac{\omega_{k} t}{2}
\end{aligned}
$$

where $\operatorname{sinc}(x) \equiv \sin (x) / x$.

Before proceeding to calculate the Lamb shift and decoherence parameters, we should note that in the definition of $\mathbf{S}_{\alpha}$ and $\mathbf{B}_{\alpha}$ in Eq. (3.1), each $\mathbf{S}_{\alpha}$ is coupled to a $\mathbf{B}_{\alpha}$ with the same index $\alpha$, whereas in the present case each $\mathbf{S}_{\alpha}$ (i.e., $\sigma_{z}$ ) is coupled to both $\mathbf{B}_{\alpha}$ (i.e., $\mathbf{b}_{k}$ ) and $\mathbf{B}_{\alpha}^{\dagger}$. Let us briefly again suppress for clarity the $i j, k l$ indices of the present example. By linearity, the required modification is clearly that Eq. (3.16) should be replaced with

$$
\begin{aligned}
b_{\mu \nu, \alpha}(t) & =-i t \sqrt{\wp_{\mu}} \sum_{\alpha^{\prime} \alpha^{\prime \prime}}\left[\lambda_{\alpha^{\prime}}\left\langle\mu\left|\mathbf{B}_{\alpha^{\prime \prime}}\right| \nu\right\rangle \Gamma_{\alpha^{\prime}}^{\alpha \alpha^{\prime \prime}}(t)\right. \\
& \left.+\lambda_{\alpha^{\prime}}^{*}\left\langle\mu\left|\mathbf{B}_{\alpha^{\prime \prime}}^{\dagger}\right| \nu\right\rangle\left(\Gamma_{\alpha^{\prime}}^{\alpha \alpha^{\prime \prime}}(t)\right)^{*}\right] \quad \alpha \geq 1
\end{aligned}
$$

(where it is assumed that the expansion coefficients of $\mathbf{S}_{\alpha}$, the $p_{\alpha \beta}(t)$, are real, as in the example treated here). Using Eqs. (3.17), (3.22) this leads to Lamb shift parameters of the form:

$$
\phi_{\alpha}=2 \sum_{\alpha^{\prime} \alpha^{\prime \prime}} \operatorname{Re}\left[\lambda_{\alpha^{\prime}}\left\langle\mathbf{B}_{\alpha^{\prime \prime}}\right\rangle_{B} \Gamma_{\alpha^{\prime}}^{\alpha \alpha^{\prime \prime}}(\tau)\right]=0
$$

since using Eq. (4.4) expectation values of creation and annihilation operators between number states vanish. As for the decoherence part, using Eq. (4.4) again we find that $\left\langle\mathbf{B}_{\alpha^{\prime \prime}} \mathbf{B}_{\beta^{\prime \prime}}\right\rangle_{B}$ and $\left\langle\mathbf{B}_{\alpha^{\prime \prime}}^{\dagger} \mathbf{B}_{\beta^{\prime \prime}}^{\dagger}\right\rangle_{B}$ vanish, so that using Eqs. (3.19), (3.23), the decoherence parameters are:

$$
\begin{aligned}
\left\langle\dot{\chi}_{\alpha \beta}\right\rangle= & \tau \sum_{\alpha^{\prime} \alpha^{\prime \prime} \beta^{\prime} \beta^{\prime \prime}} \lambda_{\alpha^{\prime}} \lambda_{\beta^{\prime}}^{*}\left[\left\langle\mathbf{B}_{\alpha^{\prime \prime}} \mathbf{B}_{\beta^{\prime \prime}}^{\dagger}\right\rangle_{B} \Gamma_{\alpha^{\prime}}^{\alpha \alpha^{\prime \prime}}(\tau) \Gamma_{\beta^{\prime}}^{\beta \beta^{\prime \prime}}(\tau)^{*}\right. \\
& \left.+\left\langle\mathbf{B}_{\alpha^{\prime \prime}}^{\dagger} \mathbf{B}_{\beta^{\prime \prime}}\right\rangle_{B} \Gamma_{\alpha^{\prime}}^{\alpha \alpha^{\prime \prime}}(\tau)^{*} \Gamma_{\beta^{\prime}}^{\beta \beta^{\prime \prime}}(\tau)\right],
\end{aligned}
$$

or, using the results for the spin-boson case: 


$$
\begin{aligned}
\left\langle\dot{\chi}_{i \xi, j \xi_{1}}\right\rangle= & \tau \sum_{i^{\prime} j^{\prime}, k^{\prime} l^{\prime} ; k^{\prime \prime} l^{\prime \prime}} \lambda_{k^{\prime}}^{i^{\prime}} \lambda_{l^{\prime}}^{j^{\prime} *} \times \\
& {\left[\left\langle\mathbf{b}_{k^{\prime \prime}} \mathbf{b}_{l^{\prime \prime}}^{\dagger}\right\rangle_{B} \Gamma_{i^{\prime} z, k^{\prime}}^{i \xi k^{\prime \prime}}(\tau)\left(\Gamma_{j^{\prime} z, l^{\prime}}^{j \xi_{1} ; l^{\prime \prime}}(\tau)\right)^{*}\right.} \\
& \left.+\left\langle\mathbf{b}_{k^{\prime \prime}}^{\dagger} \mathbf{b}_{l^{\prime \prime}}\right\rangle_{B}\left(\Gamma_{i^{\prime} z, k^{\prime}}^{i \xi ; k^{\prime \prime}}(\tau)\right)^{*} \Gamma_{j^{\prime} z, l^{\prime}}^{j \xi_{1} ; l^{\prime \prime}}(\tau)\right] \\
= & \tau \delta_{\xi z} \delta_{\xi_{1} z} \sum_{k} \lambda_{k}^{i} \lambda_{k}^{j *} \operatorname{sinc}^{2}\left(\omega_{k} \tau / 2\right) \operatorname{coth} \frac{\beta \hbar \omega_{k}}{2} \\
= & a_{i j}^{z z} .
\end{aligned}
$$

Our final result for the SME in the interaction picture can thus be written as

$$
\frac{\partial \rho_{I}(t)}{\partial t}=\frac{1}{2} \sum_{i, j} a_{i j}^{z z}\left(\left[\sigma_{z}^{i}, \rho_{I}(t) \sigma_{z}^{j}\right]+\left[\sigma_{z}^{i} \rho_{I}(t), \sigma_{z}^{j}\right]\right),
$$

where

$$
a_{i j}^{z z}=\frac{\tau}{\hbar^{2}} \sum_{k} \lambda_{k}^{i} \lambda_{k}^{j *} \operatorname{sinc}^{2}\left(\omega_{k} \tau / 2\right) \operatorname{coth} \frac{\beta \hbar \omega_{k}}{2} .
$$

and we reintroduced $\hbar$.

As commented above, the dependence on $\tau$ should disappear after all is done. Let us see how this comes about within a simple continuum model. If we assume that $\left|\lambda_{k}\right|^{2}$ only depends on $\omega_{k}$, we can rewrite this expression as an integral over $\omega$ :

$$
\begin{aligned}
a_{i j}(\tau)= & \frac{1}{\hbar^{2}} \int_{0}^{\infty} d \omega g(\omega) \lambda^{i}(\omega) \lambda^{j *}(\omega) \times \\
& \tau \operatorname{sinc}^{2} \frac{\omega \tau}{2} \operatorname{coth} \frac{\beta \hbar \omega}{2},
\end{aligned}
$$

where $g(\omega)$ is the density of states. In any reasonable physical model the density of states has a cut-off frequency $\omega_{c}$. Now, $\tau \operatorname{sinc}^{2}(\omega \tau / 2)$ is a function that peaks strongly at 0 , has a width $1 / \tau$ and its integral is finite: $\frac{1}{\pi} \int_{0}^{\infty} d \omega \tau \operatorname{sinc}^{2}(\omega \tau / 2)=1$, i.e.,

$$
\begin{aligned}
\tilde{\delta}(\tau, \omega) & \equiv \frac{1}{\pi} \tau \operatorname{sinc}^{2}(\omega \tau / 2) \\
\lim _{\tau=\infty} \tilde{\delta}(\tau, \omega) & =\delta(\omega)
\end{aligned}
$$

But $\tau$, the coarse-graining timescale, must indeed be large compared to the timescale of the bath $\tau_{c}=1 / \omega_{c}$ [recall Eq. (2.14)], so in this limit we can perform the integral and we finally get:

$$
a_{i j}^{z z}=\frac{\pi}{\hbar^{2}} \lim _{\omega=0} g(\omega) \lambda^{i}(\omega) \lambda^{j}(\omega)^{*} \operatorname{coth} \frac{\beta \hbar \omega}{2} .
$$

Thus, the dependence on $\tau$ has indeed disappeared.

We now apply this result to the case of a single twolevel atom coupled to a harmonic bath. In the case of phonons and electromagnetic radiation, the interaction couples to the amplitude of the oscillators: $\mathbf{x}=$ $\sqrt{\hbar /(2 m \omega)}\left(\mathbf{b}^{\dagger}+\mathbf{b}\right)$, so that $|\lambda(\omega)|^{2} \propto 1 / \omega$. At the relevant low-frequency regime we can equivalently use the high-temperature result:

$$
\left\langle\mathbf{b}^{\dagger} \mathbf{b}\right\rangle_{B}=\left\langle\mathbf{b b}^{\dagger}\right\rangle_{B} \approx \frac{k T}{\hbar \omega} .
$$

For a three-dimensional crystal (or radiation field) $g(\omega) \propto \omega^{2}$. Collecting terms, we see that the limit yielding $a^{z z}$ is well-defined. Decoherence depends quadratically on the coupling, and linearly on temperature and on the density of low-frequency phonons.

\section{B. Model with Dissipation}

We now generalize our model to include dissipative terms. On the other hand, to keep the analysis tractable, we will consider the case of a single spin coupled to a boson bath. We keep the system and bath Hamiltonians of Eqs. (4.1), (4.2). The new interaction Hamiltonian is:

$$
\begin{array}{r}
\mathbf{H}_{I}=\sum_{k} \sigma_{z} \otimes\left(\lambda_{k z} \mathbf{b}_{k}+\lambda_{k z}^{*} \mathbf{b}_{k}^{\dagger}\right) \\
+\sigma_{+} \otimes\left(\lambda_{k+} \mathbf{b}_{k}+\lambda_{k+}^{*} \mathbf{b}_{k}^{\dagger}\right) \\
+\sigma_{-} \otimes\left(\lambda_{k-} \mathbf{b}_{k}+\lambda_{k-}^{*} \mathbf{b}_{k}^{\dagger}\right)
\end{array}
$$

(where $\lambda_{k+}=\lambda_{k-}^{*}$ ). Transforming to the IP we find: $\sigma_{\alpha}(t)=\sigma_{\alpha} e^{i \omega_{0 \alpha} t}$ and $\mathbf{b}_{k}(t)=\mathbf{b}_{k} e^{i \omega_{k} t}$ where $\alpha=z, \pm$, and $\omega_{0 z}=0, \omega_{0 \pm}=\mp \omega_{0}$. As above, this translates into diagonal $p$ and $q$ [recall Eqs. (3.4), (3.5)]:

$$
p_{\alpha \beta}(t)=\delta_{\alpha \beta} e^{i \omega_{0 \alpha} t} \quad q_{k k^{\prime}}(t)=\delta_{k k^{\prime}} e^{i \omega_{k} t},
$$

and we find for $a_{\alpha \beta}$ :

$$
\begin{aligned}
a_{\alpha \beta}(\tau)= & \frac{\tau}{\hbar^{2}} \sum_{k} \lambda_{k \alpha} \lambda_{k \beta}^{*}\left\langle\mathbf{b}_{k}^{\dagger} \mathbf{b}_{k}\right\rangle_{B} \Gamma\left(\omega_{0 \alpha}+\omega_{k}\right) \Gamma\left(-\omega_{0 \beta}-\omega_{k}\right) \\
& +\lambda_{k \alpha^{\prime}}^{*} \lambda_{k \beta^{\prime}}\left\langle\mathbf{b}_{k} \mathbf{b}_{k}^{\dagger}\right\rangle_{B} \Gamma\left(\omega_{0 \alpha}-\omega_{k}\right) \Gamma\left(-\omega_{0 \beta}+\omega_{k}\right) .
\end{aligned}
$$

Here $\lambda_{k \alpha^{\prime}}$ is the coupling coefficient for $\sigma_{\alpha}^{\dagger}$, we already dropped the vanishing $\left\langle\mathbf{b}_{k}^{\dagger} \mathbf{b}_{k}^{\dagger}\right\rangle_{B}$ and $\left\langle\mathbf{b}_{k} \mathbf{b}_{k}\right\rangle_{B}$ terms, and

$$
\Gamma(\omega)=\frac{1}{\tau} \int_{0}^{\tau} e^{i \omega t} d t=e^{i \omega \tau / 2} \operatorname{sinc}(\omega \tau / 2) .
$$

In particular, for the diagonal terms we obtain:

$$
\begin{aligned}
a_{\alpha \alpha}(\tau)= & \frac{\tau}{\hbar^{2}} \sum_{k}\left|\lambda_{k \alpha}\right|^{2}\left\langle\mathbf{b}_{k}^{\dagger} \mathbf{b}_{k}\right\rangle_{B}\left|\Gamma\left(\omega_{0 \alpha}+\omega_{k}\right)\right|^{2} \\
& +\left|\lambda_{k \alpha^{\prime}}\right|^{2}\left\langle\mathbf{b}_{k} \mathbf{b}_{k}^{\dagger}\right\rangle_{B}\left|\Gamma\left(\omega_{0 \alpha}-\omega_{k}\right)\right|^{2} .
\end{aligned}
$$

For $a_{z z}$ this yields the same result as above. For the new decoherence parameters $a_{++}$and $a_{--}$we find: 


$$
\begin{aligned}
a_{++}(\tau)= & \frac{\tau}{\hbar^{2}} \sum_{k}\left|\lambda_{k+}\right|^{2}\left(\left\langle\mathbf{b}_{k}^{\dagger} \mathbf{b}_{k}\right\rangle \operatorname{sinc}^{2}\left(\left(\omega_{k}-\omega_{0}\right) \tau / 2\right)\right. \\
& \left.+\left\langle\mathbf{b}_{k} \mathbf{b}_{k}^{\dagger}\right\rangle \operatorname{sinc}^{2}\left(\left(\omega_{k}+\omega_{0}\right) \tau / 2\right)\right) \\
a_{--}(\tau)= & \frac{\tau}{\hbar^{2}} \sum_{k}\left|\lambda_{k-}\right|^{2}\left(\left\langle\mathbf{b}_{k}^{\dagger} \mathbf{b}_{k}\right\rangle \operatorname{sinc}^{2}\left(\left(\omega_{k}+\omega_{0}\right) \tau / 2\right)\right. \\
& \left.+\left\langle\mathbf{b}_{k} \mathbf{b}_{k}^{\dagger}\right\rangle \operatorname{sinc}^{2}\left(\left(\omega_{k}-\omega_{0}\right) \tau / 2\right),\right)
\end{aligned}
$$

or, using the integral form:

$$
\begin{aligned}
a_{++}(\tau)= & \frac{\pi}{\hbar^{2}} \int_{0}^{\infty} d \omega g(\omega)\left|\lambda_{+}(\omega)\right|^{2} \times \\
& \left(\left\langle\mathbf{b}^{\dagger} \mathbf{b}\right\rangle \tilde{\delta}\left(\omega-\omega_{0}\right)+\left\langle\mathbf{b b}^{\dagger}\right\rangle \tilde{\delta}\left(\omega+\omega_{0}\right)\right) \\
a_{--}(\tau)= & \frac{\pi}{\hbar^{2}} \int_{0}^{\infty} d \omega g(\omega)\left|\lambda_{-}(\omega)\right|^{2} \times \\
& \left(\left\langle\mathbf{b}^{\dagger} \mathbf{b}\right\rangle \tilde{\delta}\left(\omega+\omega_{0}\right)+\left\langle\mathbf{b} \mathbf{b}^{\dagger}\right\rangle \tilde{\delta}\left(\omega-\omega_{0}\right)\right)
\end{aligned}
$$

With the appearance of the system's unitary time-scale $\left(1 / \omega_{0}\right)$ we have to redefine our coarse-graining procedure. We can consider two opposite limits, where either i) the system energy, or ii) the interaction energy is dominant. These two limits correspond respectively to i) the system's internal unitary evolution being fast (so we are actually coarse-graining this out as well), and ii) the system's unitary evolution being slow.

\section{The fast system limit}

In this case $\omega_{0} \tau \gg 1$. The $\delta$ functions are centered at $\omega_{0}$ and at $-\omega_{0}$, much further from zero than their width. The ones at $-\omega_{0}$ thus do not contribute (off-resonance), so, similarly to $a_{z z}$ we find:

$$
\begin{aligned}
& a_{++}=\frac{2 \pi}{\hbar^{2}} \lim _{\omega=\omega_{0}} g(\omega)\left|\lambda_{+}(\omega)\right|^{2}\left\langle\mathbf{b}^{\dagger} \mathbf{b}\right\rangle \\
& a_{--}=\frac{2 \pi}{\hbar^{2}} \lim _{\omega=\omega_{0}} g(\omega)\left|\lambda_{-}(\omega)\right|^{2}\left\langle\mathbf{b b}^{\dagger}\right\rangle .
\end{aligned}
$$

The off-diagonal Lindblad parameters $a_{\alpha \beta}$ vanish, as they involve the product of two $\delta$ functions that are centered further apart than their widths. The coefficient matrix is diagonal in the fast system limit. If we assume for further simplicity that $\lambda_{+}(\omega)=\lambda_{-}(\omega)$, then the diagonal parameters $a_{++}$and $a_{--}$only differ in the bath expectation values at $\omega_{0}$. It then follows that

$$
a_{--}=a_{++} e^{\beta \hbar \omega_{0}} .
$$

Let us now consider briefly the resulting interactionpicture master equation. Using the notation $a=a_{z z}, b=$ $a_{++}, c=a_{--}$for simplicity, we obtain:

$$
\frac{d \rho}{d t}=\left(\begin{array}{cc}
c \rho_{11}-b \rho_{00} & -\left(2 a+\frac{b+c}{2}\right) \rho_{01} \\
-\left(2 a+\frac{b+c}{2}\right) \rho_{10} & b \rho_{00}-c \rho_{11}
\end{array}\right)
$$

The off-diagonal elements decay exponentially, with a rate $\tau_{\mathrm{dec}}^{-1}=\left(2 a+\frac{b+c}{2}\right)$. The diagonal elements approach the thermal equilibrium values $\rho_{00}^{\text {ther }}$ and $\rho_{11}^{\text {ther }}$, where

$$
\frac{\rho_{00}^{\mathrm{ther}}}{\rho_{11}^{\mathrm{ther}}}=\frac{c}{b}=\frac{a_{--}}{a_{++}}=e^{\beta \hbar \omega_{0}} .
$$

The exponential rate of convergence of the diagonal elements, i.e., the dissipation rate, is $\tau_{\text {diss }}^{-1}=a_{--}+a_{++}$. Within the framework of our model, both rates depend linearly on temperature and quadratically on the corresponding coupling strengths. The important difference between them is the presence of $a_{z z}$ in the dephasing rate. The parameters $a_{++}$and $a_{--}$depend on the bath's density of states at $\omega_{0}$. Dissipation therefore can be quite slow in a number of important cases, for example when there is a gap in the phonon spectrum, or when $\omega_{0}$ is actually greater than the cutoff frequency. In these cases only much weaker multi-phonon processes cause dissipation.

The parameter $a_{z z}$, on the other hand, depends on the density of low-frequency phonons. This can be small only in very special circumstances (e.g., superfluidity, or a discrete phonon density of states as would be found in a quantum dot [23]) and its vanishing indeed usually causes macroscopic quantum-effects. In typical situations the rate of dephasing will be greater than the rate of dissipation.

The important general conclusion is the following: If our coarse-graining includes the (fast) system as well, then the density matrix rapidly decoheres into the system's energy eigen-basis 24. Then, (typically slower) it converges into the thermalized density matrix (which is of course also diagonal in the system's energy eigen-basis). See [25] for a more detailed discussion of these different regimes.

\section{The slow system limit}

In this case $\omega_{0} \tau \ll 1$. We consider only the zeroth approximation, i.e., set $\omega_{0}=0$. Using Eq. 4.18 we obtain:

$$
\begin{aligned}
a_{\alpha \beta}(\tau)= & \frac{\tau}{\hbar^{2}} \sum_{k}\left(\lambda_{k \alpha} \lambda_{k \beta}^{*}\left\langle\mathbf{b}_{k}^{\dagger} \mathbf{b}_{k}\right\rangle\right. \\
& \left.+\lambda_{k \alpha}^{*} \lambda_{k \beta}\left\langle\mathbf{b}_{k} \mathbf{b}_{k}^{\dagger}\right\rangle\right)\left|\Gamma\left(\omega_{k}\right)\right|^{2},
\end{aligned}
$$

or, integrating out the $\delta$ function again, using Eq. 4.16) for the present low-frequency limit, and assuming real $\lambda_{k \alpha}$ for simplicity:

$$
\begin{aligned}
a_{\alpha \beta} & =\frac{2 \pi}{\hbar^{2}} k T \lim _{\omega=0} \frac{g(\omega)}{\hbar \omega} \lambda_{\alpha}(\omega) \lambda_{\beta}(\omega)=a_{\alpha} a_{\beta} \\
a_{\alpha} & \equiv\left(\frac{2 \pi}{\hbar^{2}} k T \lim _{\omega=0} \frac{g(\omega)}{\hbar \omega} \lambda_{\alpha}(\omega)^{2}\right)^{1 / 2}
\end{aligned}
$$

Unlike in the fast-system case, the off-diagonal elements of the coefficient matrix do not vanish. Instead, in the 
slow-system limit $a_{\alpha \beta}$ is a projection, i.e., $a_{\alpha \beta}$ is an outer product of the vector of components $\left\{a_{\alpha}\right\}$ with itself. This allows us to write the SME using just one Lindblad operator:

$$
\mathbf{G} \equiv \sum_{\alpha} a_{\alpha} \sigma_{\alpha}
$$

This course-grained interaction operator is just a linear combination of the system operators as they appear in the interaction Hamiltonian, but with the dependence on the bath degrees of freedom already averaged out. Using $\mathbf{G}$, the SME becomes:

$$
\frac{\partial \rho(t)}{\partial t}=\frac{1}{2}\left(\left[\mathbf{G}, \rho(t) \mathbf{G}^{\dagger}\right]+\left[\mathbf{G} \rho(t), \mathbf{G}^{\dagger}\right]\right)
$$

Diagonalizing $\mathbf{G}$ and transforming $\rho$ into $\mathbf{G}$ 's eigenbasis then leads to uncoupled equations for the components of the transformed $\rho$. Therefore, in the slow system limit, the density matrix becomes diagonal in the eigenbasis of the course-grained interaction Hamiltonian (i.e., G), and for the rate of this decoherence we find:

$$
\tau_{\mathrm{dec}}^{-1}=\frac{2 \pi}{\hbar^{2}} k T \lim _{\omega=0}\left(\frac{g(\omega)}{\omega}\left(2 \lambda_{z}^{2}(\omega)+\lambda_{+}^{2}(\omega)+\lambda_{-}^{2}(\omega)\right)\right) .
$$

\section{Comparison of the Markovian Result to Exact Solution of Spin-Boson Model for Pure Dephasing}

The spin-boson model is exactly solvable in the pure dephasing limit, and we present the detailed solution in Appendix A. The result for an initial thermal bath is that the time-dependence of the off-diagonal terms is proportional to $e^{-\Gamma(T, t)}$, with

$$
\Gamma=\frac{2 t^{2}}{\hbar^{2}} \sum_{k}\left|\lambda_{k}\right|^{2} \operatorname{sinc}^{2}\left(\omega_{k} t / 2\right) \operatorname{coth} \frac{\hbar \omega_{k}}{2 k_{B} T}
$$

This exact result holds for arbitrary times $t$ and for both finite and infinite baths.

On the other hand, recall that the SME result for the single qubit case was [Eq. (4.12)]:

$$
a_{z z}=\frac{\tau}{\hbar^{2}} \sum_{k}\left|\lambda_{k}\right|^{2} \operatorname{sinc}^{2}\left(\omega_{k} \tau / 2\right) \operatorname{coth} \frac{\hbar \omega_{k}}{2 k_{B} T}
$$

This is the dephasing rate for a single qubit satisfying the Lindblad master equation

$$
\frac{d \rho}{d t}=\frac{1}{2} a_{z z}\left(\left[\sigma_{z} \rho, \sigma_{z}\right]+\left[\sigma_{z}, \rho \sigma_{z}\right]\right)
$$

whence the off-diagonal $\rho_{01} \propto \exp \left(-2 a_{z z} t\right)$.

How do these results relate to one another? We have in the Markovian case:

$$
\rho_{01}^{\mathrm{SME}} \propto \exp \left(-t \frac{2 \pi}{\hbar^{2}} \sum_{k}\left|\lambda_{k}\right|^{2} \tilde{\delta}\left(\tau, \omega_{k}\right) \operatorname{coth} \frac{\hbar \omega_{k}}{2 k_{B} T}\right)
$$

whereas in the exact case:

$$
\rho_{01}^{\text {exact }} \propto \exp \left(-t \frac{2 \pi}{\hbar^{2}} \sum_{k}\left|\lambda_{k}\right|^{2} \tilde{\delta}\left(t, \omega_{k}\right) \operatorname{coth} \frac{\hbar \omega_{k}}{2 k_{B} T}\right) .
$$

While superficially the similarity between these results is striking, there is nevertheless a crucial difference: The exact solution has recurrences, since its time-dependence is periodic (for a finite bath), whereas the SME result is a purely exponential decay. Thus they describe very different behaviors. Indeed, for small $t\left(\omega_{k} t \ll 1\right)$ the exact result decays as $\exp \left(-t^{2}\right)$ (quantum Zeno effect [26,27]), while the Markovian result always decays as $\exp (-t)$. This is of course not a surprise: the Markovian result cannot describe the dynamics for times shorter than the coarse-graining time-scale, $\tau$.

Let us now turn to see the limit in which the two solutions do agree. To prevent recurrences in the exact solution we once again replace the sum over modes by an integral, to obtain:

$$
\begin{aligned}
& \rho_{01}^{\mathrm{SME}} \propto \exp \left(-t \frac{2 \pi}{\hbar^{2}} \int d \omega g(\omega)|\lambda(\omega)|^{2} \tilde{\delta}(\tau, \omega) \operatorname{coth} \frac{\hbar \omega}{2 k_{B} T}\right) \\
& \rho_{01}^{\mathrm{exact}} \propto \exp \left(-t \frac{2 \pi}{\hbar^{2}} \int d \omega g(\omega)|\lambda(\omega)|^{2} \tilde{\delta}(t, \omega) \operatorname{coth} \frac{\hbar \omega}{2 k_{B} T}\right) .
\end{aligned}
$$

The only difference is the appearance of $\tau$ and $t$ in the widths of the $\tilde{\delta}$ functions. Now, our coarse-graining procedure was defined such that $\tau \gg 1 / \omega_{c}$ [recall the discussion surrounding Eq. (4.14)], and in this limit $\tilde{\delta}(\tau, \omega)=$ $\delta(\omega)$. For times $t>\tau, \tilde{\delta}(t, \omega)=\delta(\omega)$ also holds, so we can summarize the condition for the exact and Markovian solutions to agree as:

$$
t>\tau \gg 1 / \omega_{c}
$$

To illustrate this let us consider the Debye model as a simple example. Then:

$$
g(\omega) \propto\left\{\begin{array}{c}
\omega^{2} \text { for } \omega<\omega_{c} \\
0 \text { for } \omega \geq \omega_{c}
\end{array} .\right.
$$

As before, let the coupling coefficient $\lambda$ depend on $\omega$ only due to amplitude-coupling: $|\lambda(\omega)|^{2} \propto \omega^{-1}$. In the hightemperature $\operatorname{limit} \operatorname{coth}\left(\frac{\hbar \omega}{2 k_{B} T}\right) \propto \omega^{-1}$, so that in all we have

$$
\begin{aligned}
& \rho_{01}^{\mathrm{SME}} \propto \exp \left(-C t \tau \int_{0}^{\omega_{c}} d \omega \operatorname{sinc}^{2}(\omega \tau / 2)\right) \\
& \rho_{01}^{\text {exact }} \propto \exp \left(-C t^{2} \int_{0}^{\omega_{c}} d \omega \operatorname{sinc}^{2}(\omega t / 2)\right),
\end{aligned}
$$


where $C$ is the temperature-dependent couplingstrength, with dimensions of frequency. Figure 1 shows the argument of the exponential, $\Gamma(t)$, for the exact solution and for the SME results, corresponding to different values of the course-graining time-scale, $\tau$. The curves corresponding to the SME solutions of course are just straight lines, as they all describe simple exponential decays. It is clear that the SME solutions cannot account for the initial transition period, but for sufficiently large $\tau$ (in units of the bath cut-off time $1 / \omega_{c}$ ) the SME result approximates the exact solution very well at large times, in accordance with Eq. (4.37).

Let us summarize: The Markovian approximation we introduced gives reliable results for times greater than the course-graining time-scale, which in turn must be greater than the bath cut-off time. It does not account for the initial (transitional) time evolution, and it should be applied in cases of an infinite bath with continuous spectrum.

\section{The Lamb Shift}

Finally, in the exact solution for multiple qubits there is also a non-vanishing Lamb shift, which arises as a consequence of the Hamiltonian not commuting with itself at different times [28]. The Lamb shift does vanish for a single qubit in the exact solution of the pure dephasing spin-boson model (see Appendix A and [28]). The Lamb shift also vanished in our Markovian calculation. This discrepancy is not only due to the fact that we considered a single qubit: the more fundamental reason is that we only carried out our Markovian calculations to first order in perturbation theory, where time-ordering did not play a role. However, when we consider the multiple-qubit case in second-order perturbation theory (recall Section IIID) there is a Lamb-shift. This arises because of terms like $\sigma_{z}^{i} \sigma_{z}^{j} b_{k}^{\dagger} b_{k}$. Physically, this is a phonon-induced, indirect, exchange-interaction between the two spins. It is quadratic in $\lambda$, linear in temperature, and acts to pull the spin-energies towards an average value.

\section{CONCLUSIONS}

A central task of modern condensed phase chemistry and physics is the quantitative description of open quantum systems. These are systems that are coupled to an external uncontrollable environment (bath), a coupling which generally leads to decoherence. In this paper we provided such a quantitative description, by deriving a practical way to calculate the coefficients in the quantum Markovian semigroup master equation (commonly known as the Lindblad equation). Our starting point was the exact Kraus operator sum representation, which presents the evolution of an open quantum system as a general, completely positive, linear map. By coarsegraining this evolution over a time-scale typical of the bath (the inverse of the bath density-of-states frequencycutoff), we showed how the Lindblad equation can be derived, and how its coefficients can be systematically calculated using perturbation theory in the system-bath coupling strength. This resolves an important shortcoming in the theory of open quantum systems: so far no practical general method was known which takes as input an interaction Hamiltonian, and then produces the Lindblad equation together with all its coefficients. The complexity of our method is determined by the difficulty of calculating certain time-ordered integrals, which of course increases with higher orders of perturbation theory. In principle, this is equivalent to the calculation of standard Feynman diagrams, and thus the arsenal of techniques known in many-body physics could be employed here as well. To test the validity of our theory, we compared it here to an exactly solvable model, namely, the spin-boson Hamiltonian with pure phase-damping. For times longer than the coarse-graining time, the agreement was found to be excellent already at the level of first order perturbation theory.

\section{ACKNOWLEDGEMENTS}

It is a pleasure to acknowledge very insightful discussions with Dave Bacon. This work was supported in part by the National Security Agency (NSA) and Advanced Research and Development Activity (ARDA) under Army Research Office (ARO) contract number DAAG55-98-1-0371.

\section{APPENDIX A: ANALYTICAL SOLUTION OF THE SPIN BOSON MODEL FOR PURE DEPHASING}

We present here the analytical solution of the spinboson model for pure dephasing. The derivation is based on [28].

Starting from the interaction picture Hamiltonian:

$$
\mathbf{H}_{I}(t)=\sum_{i, k} \sigma_{z}^{i} \otimes\left[\lambda_{k}^{i} e^{-i \omega_{k} t} \mathbf{a}_{k}+\left(\lambda_{k}^{i}\right)^{*} e^{i \omega_{k} t} \mathbf{a}_{k}^{\dagger}\right]
$$

we want to find the system density matrix

$$
\rho_{I}(t)=\operatorname{Tr}_{B}\left[\rho_{\text {tot }, I}(t)\right]=\operatorname{Tr}_{B}\left[\mathbf{U}(t) \rho(0) \otimes \rho_{B}(0) \mathbf{U}^{\dagger}(t)\right]
$$

where

$$
\mathbf{U}(t)=\mathrm{T} \exp \left[-\frac{i}{\hbar} \int_{0}^{t} \mathbf{H}_{I}(\tau) d \tau\right]
$$




\section{Calculation of the Evolution Operator}

Note that $\mathbf{H}_{I}(t)$ does not commute with itself at different times, which is why we need the time-ordered product:

$$
\left[\mathbf{H}_{I}(t), \mathbf{H}_{I}\left(t^{\prime}\right)\right]=\sum_{i, i^{\prime} ; k} 2 i \sigma_{z}^{i} \sigma_{z}^{i^{\prime}} \otimes \operatorname{Re}\left[\lambda_{k}^{i}\left(\lambda_{k}^{i^{\prime}}\right)^{*}\right] \sin \omega_{k}\left(t-t^{\prime}\right) \mathbf{1}
$$

where we used the boson commutation relations $\left[\mathbf{a}_{k}, \mathbf{a}_{l}^{\dagger}\right]=\mathbf{1} \delta_{k l},\left[\mathbf{a}_{k}, \mathbf{a}_{l}\right]=0$. Note that further,

$$
\left[\left[\mathbf{H}_{I}(t), \mathbf{H}_{I}\left(t^{\prime}\right)\right], \mathbf{H}_{I}\left(t^{\prime \prime}\right)\right]=0 .
$$

This means that we can use the Baker-Hausdorf formula $\exp (A+B)=\exp (-[A, B] / 2) \exp (A) \exp (B)$ (valid if $[[A, B], A]=[[A, B], B]=0)$ to calculate $\mathbf{U}(t)$. To do so note the generalization

$$
\exp \left(\sum_{n} A_{n}\right)=\left(\prod_{n<n^{\prime}} \exp \left(-\frac{1}{2}\left[A_{n}, A_{n^{\prime}}\right]\right)\right)\left(\prod_{n} \exp \left(A_{n}\right)\right)
$$

which is valid if every second-order commutator vanishes. To apply this result for our case let us formally discretize the integrals and denote $\mathcal{H}_{n} \equiv-\frac{i}{\hbar} \mathbf{H}_{I}(n \Delta t)$. Then:

$$
\begin{aligned}
\mathbf{U}(t)= & \operatorname{T} \exp \left[-\frac{i}{\hbar} \int_{0}^{t} \mathbf{H}_{I}(\tau) d \tau\right]=\mathrm{T} \lim _{\Delta t \rightarrow 0} \exp \left[\sum_{n=0}^{N} \mathcal{H}_{n} \Delta t\right] \\
= & \lim _{\Delta t \rightarrow 0} \prod_{n<n^{\prime}} \exp \left(-\frac{1}{2}\left[\mathcal{H}_{n}, \mathcal{H}_{n^{\prime}}\right](\Delta t)^{2}\right) \prod_{n} \exp \left(\mathcal{H}_{n} \Delta t\right) \\
= & \lim _{\Delta t \rightarrow 0} \prod_{n<n^{\prime}}\left(1-\frac{1}{2}\left[\mathcal{H}_{n}, \mathcal{H}_{n^{\prime}}\right](\Delta t)^{2}\right) \prod_{n}\left(1-\mathcal{H}_{n} \Delta t\right) \\
= & \lim _{\Delta t \rightarrow 0}\left[1-\frac{1}{2} \sum_{n<n^{\prime}}\left[\mathcal{H}_{n}, \mathcal{H}_{n^{\prime}}\right](\Delta t)^{2}\right]\left[1-\sum_{n} \mathcal{H}_{n} \Delta t\right] \\
= & \lim _{\Delta t \rightarrow 0} \exp \left(-\frac{1}{2} \sum_{n<n^{\prime}}\left[\mathcal{H}_{n}, \mathcal{H}_{n^{\prime}}\right](\Delta t)^{2}\right) \exp \left(\sum_{n} \mathcal{H}_{n} \Delta t\right) \\
= & \exp \left[\left(-\frac{i}{\hbar}\right)^{2} \int_{0}^{t} d t_{1} \int_{0}^{t_{1}} d t_{2}\left[\mathbf{H}_{I}\left(t_{2}\right), \mathbf{H}_{I}\left(t_{1}\right)\right] \times\right. \\
& \exp \left[-\frac{i}{\hbar} \int_{0}^{t} \mathbf{H}_{I}(\tau) d \tau\right] .
\end{aligned}
$$

In the second line we explicitly invoked time-ordering by using the Campbell-Hausdorf formula to deal with the non-commuting problem; in the subsequent lines we used the re-exponentiation trick. In the final line there is no need for explicit time-ordering left, i.e., the integrals can be calculated as such. We find:

$$
-\frac{i}{\hbar} \int_{0}^{t} \mathbf{H}_{I}(\tau) d \tau=\sigma_{z}^{i} \otimes \sum_{i, k}\left(\alpha_{k}^{i}(t) \mathbf{a}_{k}^{\dagger}-\alpha_{k}^{i}(t)^{*} \mathbf{a}_{k}\right)
$$

where

$$
\alpha_{k}^{i}(t)=\frac{\left(\lambda_{k}^{i}\right)^{*}\left(e^{i \omega_{k} t}-1\right)}{\hbar \omega_{k}}
$$

Further:

$$
\int_{0}^{t} d t_{1} \int_{0}^{t_{1}} d t_{2} \sin \omega_{k}\left(t_{2}-t_{1}\right)=\frac{\sin \omega_{k} t-\omega_{k} t}{\omega_{k}^{2}}
$$

and

$$
\int_{0}^{t} d t_{1} \int_{0}^{t_{1}} d t_{2} \cos \omega_{k}\left(t_{2}-t_{1}\right)=\frac{1-\cos \omega_{k} t}{\omega_{k}^{2}}
$$

so that

$$
\begin{aligned}
f(t) \equiv- & i\left(-\frac{i}{\hbar}\right)^{2} \int_{0}^{t} d t_{1} \int_{0}^{t_{1}} d t_{2}\left[\mathbf{H}_{I}\left(t_{2}\right), \mathbf{H}_{I}\left(t_{1}\right)\right] \\
= & \sum_{i, i^{\prime} ; k} 2 \sigma_{z}^{i} \sigma_{z}^{i^{\prime}} \otimes \operatorname{Re}\left[\lambda_{k}^{i}\left(\lambda_{k}^{i^{\prime}}\right)^{*}\right] \frac{\omega_{k} t-\sin \omega_{k} t}{\left(\hbar \omega_{k}\right)^{2}} \mathbf{1} \\
= & \sum_{k} \frac{\omega_{k} t-\sin \omega_{k} t}{\left(\hbar \omega_{k}\right)^{2}}\left|\sum_{i} \lambda_{k}^{i} \sigma_{z}^{i}\right|^{2} \otimes \mathbf{1 .}
\end{aligned}
$$

Note that $f$ is an operator acting just on the system, and is a simple phase for the case of a single qubit. Since the $\mathbf{a}_{k}$ operators commute for different modes we have as our final simplified result for the evolution operator:

$$
\mathbf{U}(t)=e^{i f(t)} \prod_{i, k} \exp \left[\sigma_{z}^{i} \otimes\left(\alpha_{k}^{i}(t) \mathbf{a}_{k}-\alpha_{k}^{i}(t)^{*} \mathbf{a}_{k}^{\dagger}\right)\right] .
$$

\section{Calculation of the Density Matrix}

Now recall the definition of the coherent states. These are eigenstates of the annihilation operator:

$$
\mathbf{a}|\alpha\rangle=\alpha|\alpha\rangle \text {. }
$$

They are minimum-uncertainty states in a harmonic potential, etc. As is well known,

$$
|\alpha\rangle=e^{-|\alpha|^{2} / 2} \sum_{n=0}^{\infty} \frac{\alpha^{n}}{\sqrt{n !}}|n\rangle
$$

where $|n\rangle$ are number (Fock) states. The completeness relation for the coherent states is:

$$
\frac{1}{\pi} \int d^{2} \alpha|\alpha\rangle\langle\alpha|=1
$$

where the integration is over the entire complex plane. They are useful in our context since they are created by the displacement operator 


$$
D(\alpha) \equiv \exp \left(\alpha \mathbf{a}^{\dagger}-\alpha^{*} \mathbf{a}\right)=D(-\alpha)^{\dagger}
$$

acting on the vacuum state:

$$
D(\alpha)|\mathbf{0}\rangle=|\alpha\rangle,
$$

which is clearly related to $\mathbf{U}(t)$. We will need the result:

$$
D(\alpha) D(\beta)=\exp \frac{\alpha \beta^{*}-\alpha^{*} \beta}{2} D(\alpha+\beta),
$$

which is easily derived from $D(\alpha)=\exp \left(\alpha \mathbf{a}^{\dagger}-\alpha^{*} \mathbf{a}\right)$, $\left[\mathbf{a}, \mathbf{a}^{\dagger}\right]=1$, and the Baker-Hausdorf formula $\exp (A+$ $B)=\exp (-[A, B] / 2) \exp (A) \exp (B)$ (again, valid if $[[A, B], A]=[[A, B], B]=0)$.

Now let $R_{i k}(t) \equiv \alpha_{k}^{i}(t) \mathbf{a}_{k}^{\dagger}-\alpha_{k}^{i}(t)^{*} \mathbf{a}_{k}$ and consider $\exp \left[\sigma_{z}^{i} \otimes R_{i k}(t)\right]$ :

$$
\begin{aligned}
\exp \left[\sigma_{z} \otimes R\right]= & I_{S} \otimes \sum_{n=0}^{\infty} \frac{R^{2 n}}{(2 n) !}+\sigma_{z} \otimes \sum_{n=0}^{\infty} \frac{R^{2 n+1}}{(2 n+!) !} \\
= & I_{S} \otimes \cosh R+\sigma_{z} \otimes \sinh R \\
= & I_{S} \otimes \frac{1}{2}[D(\alpha)+D(-\alpha)] \\
& +\sigma_{z} \otimes \frac{1}{2}[D(\alpha)-D(-\alpha)] \\
= & |0\rangle\langle 0|\otimes D(\alpha)+| 1\rangle\langle 1| \otimes D(-\alpha) .
\end{aligned}
$$

This is an important result since it shows that depending on whether the field is coupled to the qubit $|0\rangle$ or $|1\rangle$ state, the field acquires a different displacement. This is the source of the dephasing the qubits undergo, since when acting on a superposition state of a qubit, the qubit and field become entangled:

$$
\begin{aligned}
& \exp \left[\sigma_{z} \otimes R\right](a|0\rangle+b|1\rangle)|\beta\rangle=a|0\rangle \otimes D(\alpha)|\beta\rangle \\
& +b|1\rangle \otimes D(-\alpha)|\beta\rangle \\
= & e^{\left(\alpha \beta^{*}-\alpha^{*} \beta\right) / 2} a|0\rangle \otimes|\alpha+\beta\rangle \\
& +e^{-\left(\alpha \beta^{*}-\alpha^{*} \beta\right) / 2} b|1\rangle \otimes|\beta-\alpha\rangle .
\end{aligned}
$$

The evolution operator can be written as:

$$
\mathbf{U}(t)=e^{i f(t)} \prod_{i, k}\left[|0\rangle_{i}\left\langle 0\left|\otimes D\left(\alpha_{k}^{i}\right)+\right| 1\right\rangle_{i}\langle 1| \otimes D\left(-\alpha_{k}^{i}\right)\right] .
$$

Now assume that the boson bath is in thermal equilibrium:

$$
\begin{aligned}
\rho_{B} & =\frac{1}{Z} e^{-\beta \mathbf{H}_{B}} \\
& =\left[\prod_{k} \frac{e^{-\beta \hbar \omega_{k} / 2}}{1-e^{-\beta \hbar \omega_{k}}}\right]^{-1} \exp \left(-\beta \sum_{k} \hbar \omega_{k}\left(\mathbf{N}_{k}+\frac{1}{2}\right)\right) \\
& =\prod_{k} \frac{1}{\left\langle\mathbf{N}_{k}\right\rangle} \exp \left(-\beta \hbar \omega_{k} \mathbf{N}_{k}\right),
\end{aligned}
$$

where the mean boson occupation number is:

$$
\left\langle\mathbf{N}_{k}\right\rangle=\frac{1}{e^{\beta \hbar \omega_{k}}-1} .
$$

As shown in [5], p.122-3, this can be transformed into the coherent-state representation, with the result:

$$
\rho_{B}=\prod_{k} \rho_{B, k}
$$

where

$$
\rho_{B, k}=\frac{1}{\pi\left\langle\mathbf{N}_{k}\right\rangle} \int d^{2} \alpha_{k} \exp \left(-\frac{\left|\alpha_{k}\right|^{2}}{\left\langle\mathbf{N}_{k}\right\rangle}\right)\left|\alpha_{k}\right\rangle\left\langle\alpha_{k}\right| .
$$

Now consider the system density matrix. Let $\rho_{x_{i}, y_{i}}=$ $|x\rangle_{i}\langle y|$ where $x, y=\{0,1\}$. Since we are dealing with qubits the system density matrix is a sum of all possible tensor products of single qubit pure states, i.e., of terms of the form $\rho_{\left\{x_{i}, y_{i}\right\}} \equiv \rho_{x_{1}, y_{1}} \otimes \cdots \otimes \rho_{x_{N}, y_{N}}$. Thus it can be expanded as

$$
\rho(0)=\sum_{\left\{x_{i}, y_{i}\right\}} c_{\left\{x_{i}, y_{i}\right\}} \rho_{\left\{x_{i}, y_{i}\right\}} .
$$

Recall that we set out to evaluate $\rho(t)=$ $\operatorname{Tr}_{B}\left[\mathbf{U}(t) \rho(0) \otimes \rho_{B}(0) \mathbf{U}^{\dagger}(t)\right]$. For simplicity let us now consider the case of a single qubit. It suffices to calculate the evolution of each of the four pure states $|x\rangle\langle y|$ separately. Thus

$$
\begin{aligned}
\rho_{x, y}(t)= & \operatorname{Tr}_{B}\left[\mathbf{U}(t)|x\rangle\langle y| \otimes \rho_{B}(0) \mathbf{U}^{\dagger}(t)\right] \\
= & \operatorname{Tr}_{B}\left[e^{i f(t)} \prod_{k}\left[|0\rangle\left\langle 0\left|\otimes D\left(\alpha_{k}\right)+\right| 1\right\rangle\langle 1| \otimes D\left(-\alpha_{k}\right)\right]\right. \\
& |x\rangle\langle y| \otimes \prod_{m} \rho_{B, m} \\
& \left.\prod_{l}\left[|0\rangle\left\langle 0\left|\otimes D^{\dagger}\left(\alpha_{l}\right)+\right| 1\right\rangle\langle 1| \otimes D^{\dagger}\left(-\alpha_{l}\right)\right] e^{-i f^{\dagger}(t)}\right] .
\end{aligned}
$$

The terms in the three products match one-to-one for equal indices, so we can write everything as a product over a single index $k$. Using $\operatorname{Tr}(A \otimes B)=\operatorname{Tr} A \times \operatorname{Tr} B$ to rearrange the order of the trace and the products, and $D^{\dagger}(-\alpha)=D(\alpha)$, we have:

$$
\begin{aligned}
\rho_{x, y}(t)= & \delta_{x, 0} \delta_{y, 0} e^{i f(t)}|0\rangle\langle 0| e^{-i f^{\dagger}(t)} \\
& \otimes \prod_{k} \operatorname{Tr}_{B}\left[D\left(\alpha_{k}\right) \rho_{B, k} D\left(-\alpha_{k}\right)\right] \\
+ & \delta_{x, 0} \delta_{y, 1} e^{i f(t)}|0\rangle\langle 1| e^{-i f^{\dagger}(t)} \\
& \otimes \prod_{k} \operatorname{Tr}_{B}\left[D\left(\alpha_{k}\right) \rho_{B, k} D\left(\alpha_{k}\right)\right] \\
+ & \delta_{x, 1} \delta_{y, 0} e^{i f(t)}|1\rangle\langle 0| e^{-i f^{\dagger}(t)} \\
& \otimes \prod_{k} \operatorname{Tr}_{B}\left[D\left(-\alpha_{k}\right) \rho_{B, k} D\left(-\alpha_{k}\right)\right] \\
+ & \delta_{x, 1} \delta_{y, 1} e^{i f(t)}|1\rangle\langle 1| e^{-i f^{\dagger}(t)} \\
& \otimes \prod_{k} \operatorname{Tr}_{B}\left[D\left(-\alpha_{k}\right) \rho_{B, k} D\left(\alpha_{k}\right)\right] .
\end{aligned}
$$


Consider the $\operatorname{Tr}_{B}$ terms: for $|0\rangle\langle 0|$ and $|1\rangle\langle 1|$ by cycling in the trace the displacement operators cancel and $\operatorname{Tr}_{B}\left[\rho_{B, k}\right]=1$. Thus, as expected the diagonal terms do not change [apart from the Lamb shift due to $f(t)$ ]. As for the off-diagonal terms (evaluating the trace in any complete basis):

$$
\begin{aligned}
& \operatorname{Tr}_{B}\left[D\left( \pm 2 \alpha_{k}\right) \rho_{B, k}\right] \\
= & \frac{1}{\pi\left\langle\mathbf{N}_{k}\right\rangle} \int d^{2} \beta_{k} \exp \left(-\frac{\left|\beta_{k}\right|^{2}}{\left\langle\mathbf{N}_{k}\right\rangle}\right) \sum_{n}\left\langle n\left|D\left( \pm 2 \alpha_{k}\right)\right| \beta_{k}\right\rangle\left\langle\beta_{k} \mid n\right\rangle \\
= & \frac{1}{\pi\left\langle\mathbf{N}_{k}\right\rangle} \int d^{2} \beta_{k} \exp \left(-\frac{\left|\beta_{k}\right|^{2}}{\left\langle\mathbf{N}_{k}\right\rangle}\right)\left\langle\beta_{k}\left|D\left( \pm 2 \alpha_{k}\right)\right| \beta_{k}\right\rangle .
\end{aligned}
$$

Now:

$$
\begin{aligned}
& \langle\beta|D( \pm 2 \alpha)| \beta\rangle \\
= & \exp \left[ \pm\left(\alpha \beta^{*}-\alpha^{*} \beta\right)\right]\langle\beta \mid \pm \alpha+\beta\rangle \\
= & \exp \left[ \pm\left(\alpha \beta^{*}-\alpha^{*} \beta\right)\right] \times \\
& \exp \left[\beta^{*}( \pm 2 \alpha+\beta)-\frac{1}{2}\left(|\beta|^{2}+| \pm 2 \alpha+\beta|^{2}\right)\right] \\
= & \exp \left(-2|\alpha|^{2} \pm 2\left(\alpha \beta^{*}-\alpha^{*} \beta\right)\right) .
\end{aligned}
$$

Thus:

$$
\begin{aligned}
& \operatorname{Tr}_{B}\left[D\left( \pm 2 \alpha_{k}\right) \rho_{B, k}\right]=\exp \left(-2\left|\alpha_{k}\right|^{2}\right) \frac{1}{\pi\left\langle\mathbf{N}_{k}\right\rangle} \times \\
& \int d^{2} \beta_{k} \exp \left(-\frac{\left|\beta_{k}\right|^{2}}{\left\langle\mathbf{N}_{k}\right\rangle} \pm 2\left(\alpha_{k} \beta_{k}^{*}-\alpha_{k}^{*} \beta_{k}\right)\right) \\
= & \frac{\exp \left(-2\left|\alpha_{k}\right|^{2}\right)}{\pi\left\langle\mathbf{N}_{k}\right\rangle}\left[\pi\left\langle\mathbf{N}_{k}\right\rangle \exp \left(-4\left|\alpha_{k}\right|^{2}\left\langle\mathbf{N}_{k}\right\rangle\right)\right] \\
= & \exp \left[-4\left|\alpha_{k}\right|^{2}\left(\left\langle\mathbf{N}_{k}\right\rangle+\frac{1}{2}\right)\right] \\
= & \exp \left[-4\left|\frac{\lambda_{k}^{*}\left(e^{i \omega_{k} t}-1\right)}{\hbar \omega_{k}}\right|^{2}\left(\frac{1}{e^{\beta \hbar \omega_{k}}-1}+\frac{1}{2}\right)\right] \\
= & \exp \left[-4\left|\lambda_{k}\right|^{2} \frac{1-\cos \omega_{k} t}{\left(\hbar \omega_{k}\right)^{2}} \operatorname{coth} \beta \hbar \omega_{k} / 2\right] .
\end{aligned}
$$

Thus decay of the off-diagonal terms goes as $e^{-\Gamma(T, t)}$, with

$$
\Gamma=4 \sum_{k}\left|\lambda_{k}\right|^{2} \frac{1-\cos \omega_{k} t}{\left(\hbar \omega_{k}\right)^{2}} \operatorname{coth} \frac{\hbar \omega_{k}}{2 k_{B} T}
$$

which is equivalent to the result that appeared in Eq.(4.32) above.

[1] E. Schrödinger, Naturwissenschaften 23, 807 (1935), translated in [2].
[2] Quantum Theory and Measurement, edited by J.A. Wheeler and W.H. Zurek (Princeton University, Princeton, NJ, 1983).

[3] E.B. Davies, Quantum Theory of Open Systems (Academic Press, London, 1976).

[4] R. Alicki and K. Lendi, Quantum Dynamical Semigroups and Applications, No. 286 in Lecture Notes in Physics (Springer-Verlag, Berlin, 1987).

[5] C. Gardiner, Quantum Noise, Vol. 56 of Springer Series in Synergetics (Springer-Verlag, Berlin, 1991).

[6] U. Weiss, Quantum Dissipative Systems (World Scientific, Singapore, 1993).

[7] W.H. Zurek, Phys. Rev. D 24, 1516 (1981).

[8] Decoherence and the Appearance of a Classical World in Quantum Theory, edited by D. Giulini, E. Joos, C. Kiefer, J. Kupsch, I.-O. Stamatescu, and H.D. Zeh (Springer-Verlag, Berlin, 1996).

[9] R. W. Zwanzig, Statistical Mechanics of Irreversibility, Vol. 3 of Lectures in Theoretical Physics (Interscience, New York, 1961), p. 106.

[10] D. Kohen, C.C. Marston and D.J. Tannor, J. Chem. Phys. 107, 5236 (1997).

[11] N. Makri, Comp. Phys. Commun. 63, 389 (1991).

[12] P. Pechukas, Phys. Rev. Lett. 73, 1060 (1994).

[13] R. Alicki, Phys. Rev. Lett. 75, 3020 (1995), Comment on "Reduced Dynamics Need Not Be Completely Positive"; Reply by P. Pechukas, ibid., p. 3021.

[14] K. Kraus, States, Effects and Operations, Fundamental Notions of Quantum Theory (Academic, Berlin, 1983).

[15] Quantum Computation and Quantum Information, M.A. Nielsen and I.L. Chuang (Cambridge University Press, Cambridge, UK, 2000).

[16] G. Lindblad, Commun. Math. Phys. 48, 119 (1976).

[17] D. Bacon, D.A. Lidar and K.B. Whaley, Phys. Rev. A 60, 1944 (1999).

[18] W.T. Pollard, A.K. Felts, and R.A. Friesner, in The Redfield equation in condensed-phase quantum dynamics, Vol. XCIII of Advances in Chemical Physics, edited by I. Prigogine and S.A. Rice (John Wiley \& Sons, New York, 1996), p. 77.

[19] P. Beck and K. Lendi, Phys. Rev. A 47, 346 (1993).

[20] T. Yu, L. Diosi, N. Gisin, and W.T. Strunz, Phys. Lett. A 265, 331 (2000).

[21] N. March, W.H. Young and S. Sampanthar, The ManyBody Problem in Quantum Mechanics (Dover, New York, 1995).

[22] A.J. Leggett, S. Chakravarty, A.T. Dorsey, M.A.P. Fisher, A. Garg and W. Zwerger, Rev. Mod. Phys. 59, 1 (1987).

[23] T. Takagahara, J. of Luminescence 70, 129 (1996).

[24] J.P. Paz and W.H. Zurek, Phys. Rev. Lett. 82, 5181 (1999).

[25] G.M. Palma, K.-A. Suominen and A.K. Ekert, Proc. Roy. Soc. London Ser. A 452, 567 (1996).

[26] B. Misra and E.C.G. Sudarshan, J. Math. Phys. 18, 756 (1977)

[27] W.M. Itano, D.J. Heinzen, J.J. Bollinger, and D.J. Wineland, Phys. Rev. A 41, 2295 (1990)

[28] L.-M. Duan and G.-C. Guo, Decoherence of quantum registers, LANL Report No. quant-ph/9703036. 


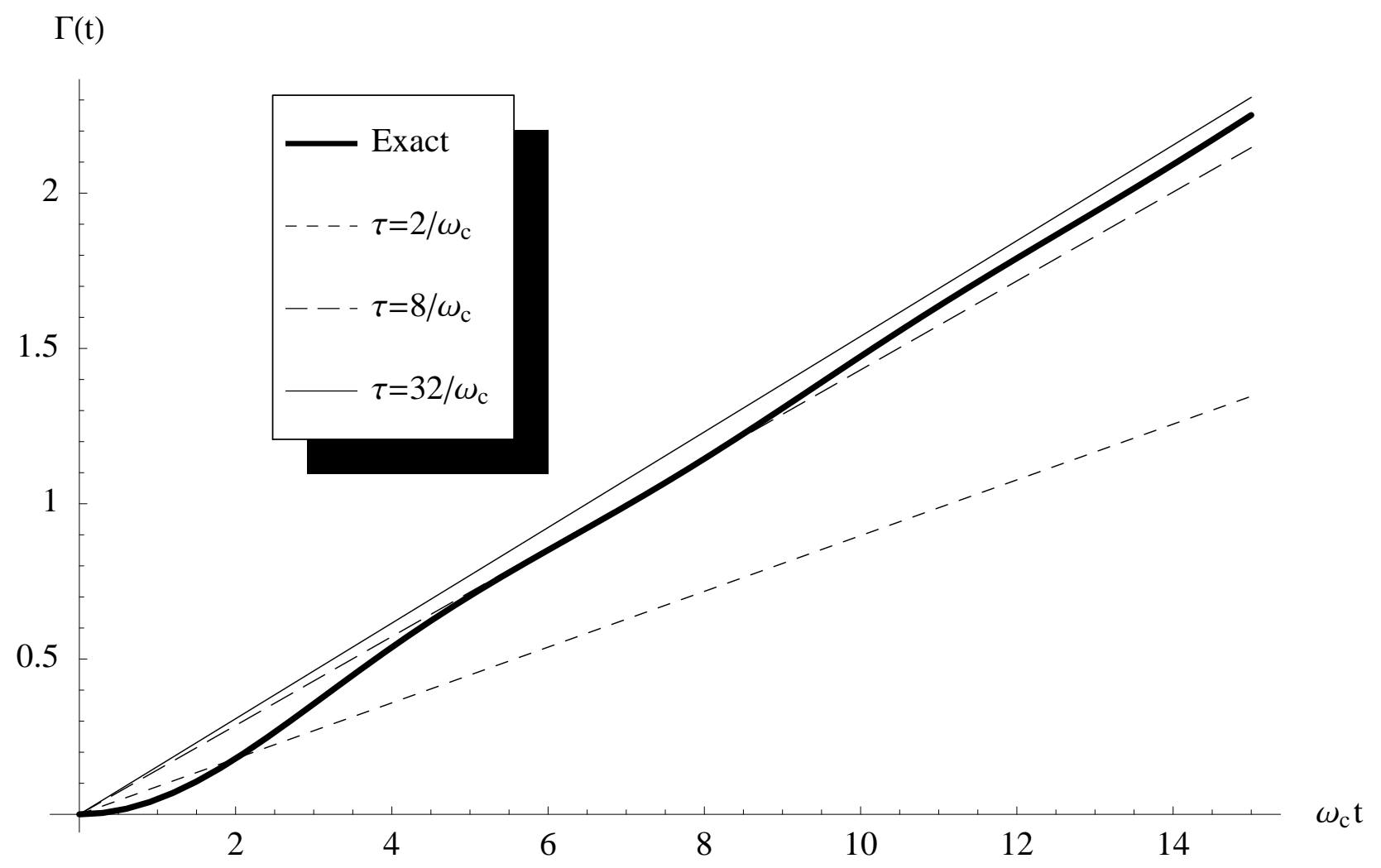

FIG. 1. Comparison of exact solution of the spin-boson model for single-qubit pure dephasing to the result obtained from the Markovian master equation. Straight lines correspond to the Markovian solution, which intersects the exact solution (thick line) at $t=\tau$, as seen from Eqs. (4.38), 4.39). The density of states of the boson bath is represented by the Debye model here. Data is plotted for $C=0.05$ and $\omega_{c}=1$. 